Supporting Information for

\title{
Sulfate-Incarcerating Nanojars: Solution and Solid State Studies, Sulfate Extraction from Water, and Anion Exchange with Carbonate
}

\author{
Basil M. Ahmed, Christian K. Hartman and Gellert Mezei* \\ Department of Chemistry, Western Michigan University, Kalamazoo, Michigan, USA \\ *Email: gellert.mezei@wmich.edu
}

CONTENTS

PAGE

1. Mass spectrometric data (Figures S1-S12) S2

2. ${ }^{1}$ H NMR spectroscopic data (Table S1, Figures S13-S24) S10

3. X-ray crystallographic data (Figures S25-S27) $\quad$ S22

$\begin{array}{ll}\text { 4. Thermogravimetric data (Figure S28) } & \text { S23 }\end{array}$ 

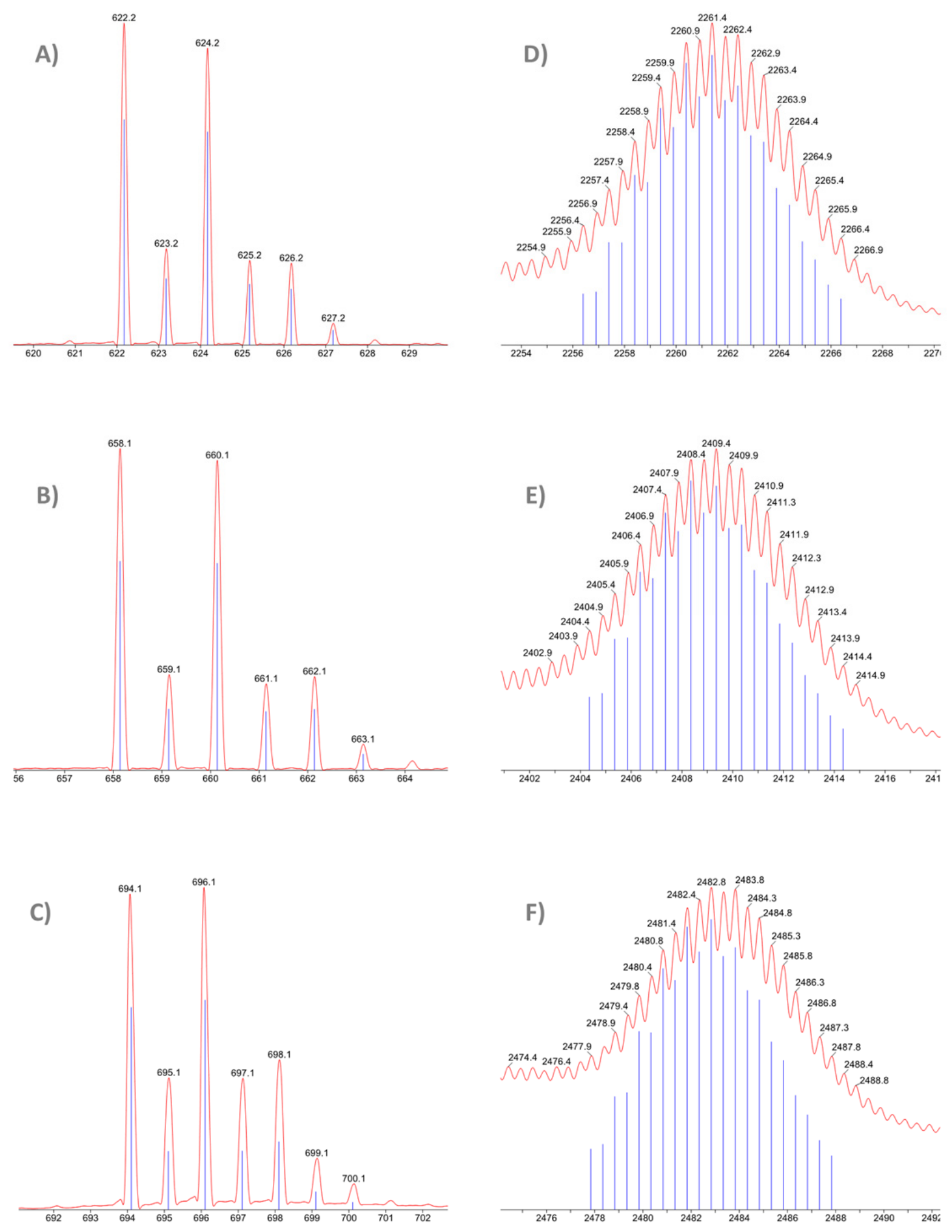

Figure S1. Predicted (blue) and observed (red) isotopic patterns for $\left[\left(\mathrm{Bu}_{4} \mathrm{~N}\right) \mathrm{Cu}_{2}(\mathrm{pz})_{2}\left(\mathrm{CO}_{3}\right)_{2}\right]^{-}$(A), $\left[\left(\mathrm{Bu}_{4} \mathrm{~N}\right) \mathrm{Cu}_{2}(\mathrm{pz})_{2}\left(\mathrm{CO}_{3}\right)\left(\mathrm{SO}_{4}\right)\right]^{-}(\mathbf{B}),\left[\left(\mathrm{Bu}_{4} \mathrm{~N}\right) \mathrm{Cu}_{2}\left(\mathrm{pz}_{2}\left(\mathrm{SO}_{4}\right)_{2}\right]^{-}(\mathbf{C}),\left[\mathrm{SO}_{4} \subset\{\mathrm{Cu}(\mathrm{OH})(\mathrm{pz})\}_{30}\right]^{2-}(\mathbf{D})\right.$, $\left[\mathrm{SO}_{4} \subset\{\mathrm{Cu}(\mathrm{OH})(\mathrm{pz})\}_{32}\right]^{2-}(\mathbf{E})$ and $\left[\mathrm{SO}_{4} \subset\{\mathrm{Cu}(\mathrm{OH})(\mathrm{pz})\}_{33}\right]^{2-}(\mathbf{F})$. 


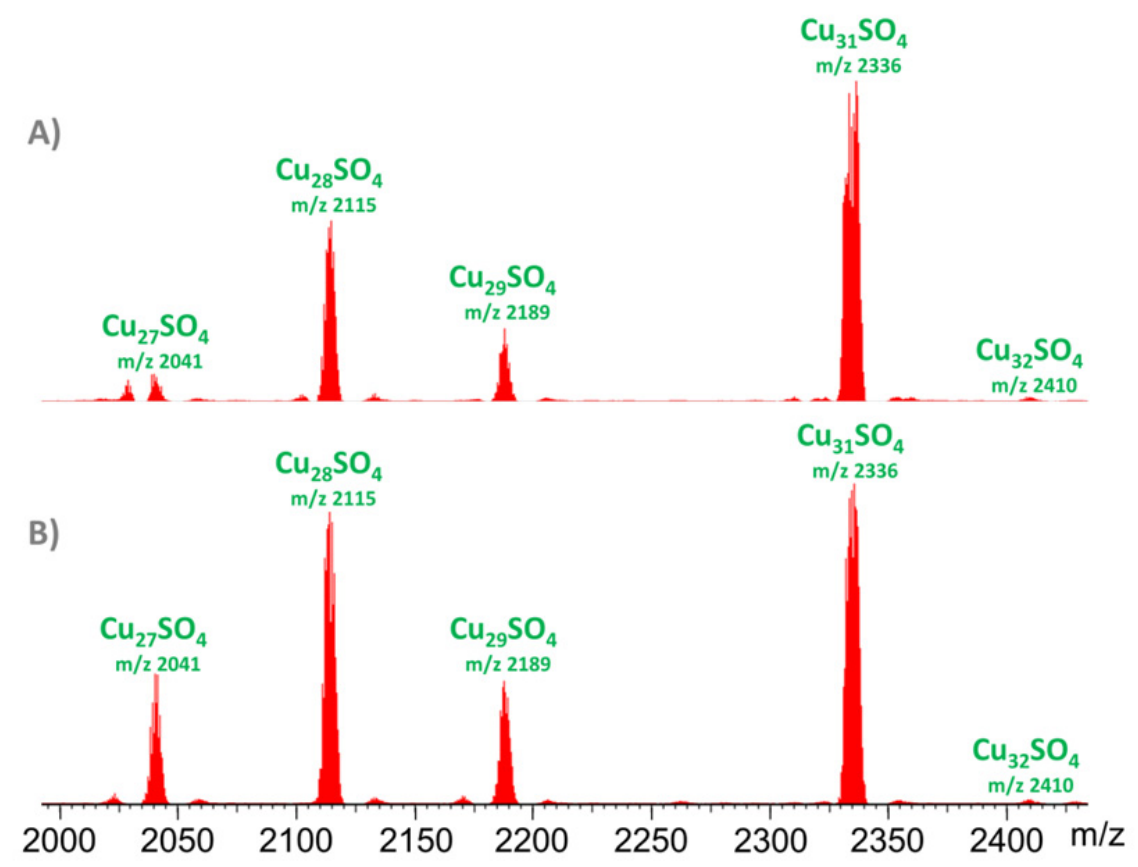

Figure S2. ESI-MS(-) spectra (in $\mathrm{CH}_{3} \mathrm{CN}$ ) of the reaction product of $\mathrm{CuSO}_{4}$, pyrazole and $\mathrm{NaOH}$ in THF with substoichiometric amount of $\mathrm{Bu}_{4} \mathrm{NOH}(\mathbf{A})$, and with excess $\mathrm{Bu}_{4} \mathrm{NOH}(\mathbf{B})$.

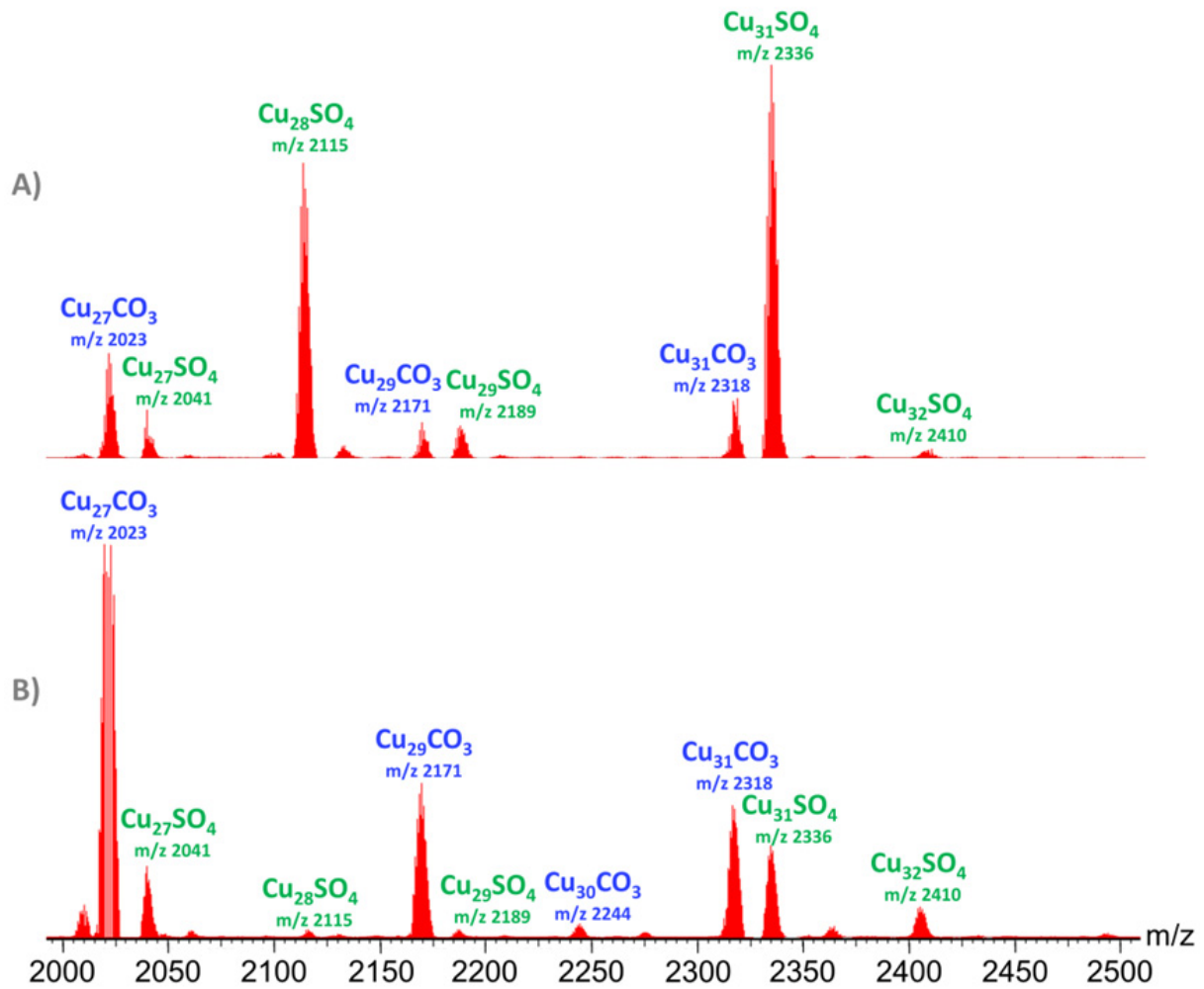

Figure S3. ESI-MS(-) spectra (in $\mathrm{CH}_{3} \mathrm{CN}$ ) of the reaction product of $\mathrm{CuSO}_{4}$, pyrazole and $\mathrm{NaOH}$ (up to $0.5 \% \mathrm{Na}_{2} \mathrm{CO}_{3}$ ) in THF (A), and $\mathrm{CuSO}_{4}$, pyrazole and $\mathrm{KOH}$ (up to $2 \% \mathrm{~K}_{2} \mathrm{CO}_{3}$ ) in THF (B). 


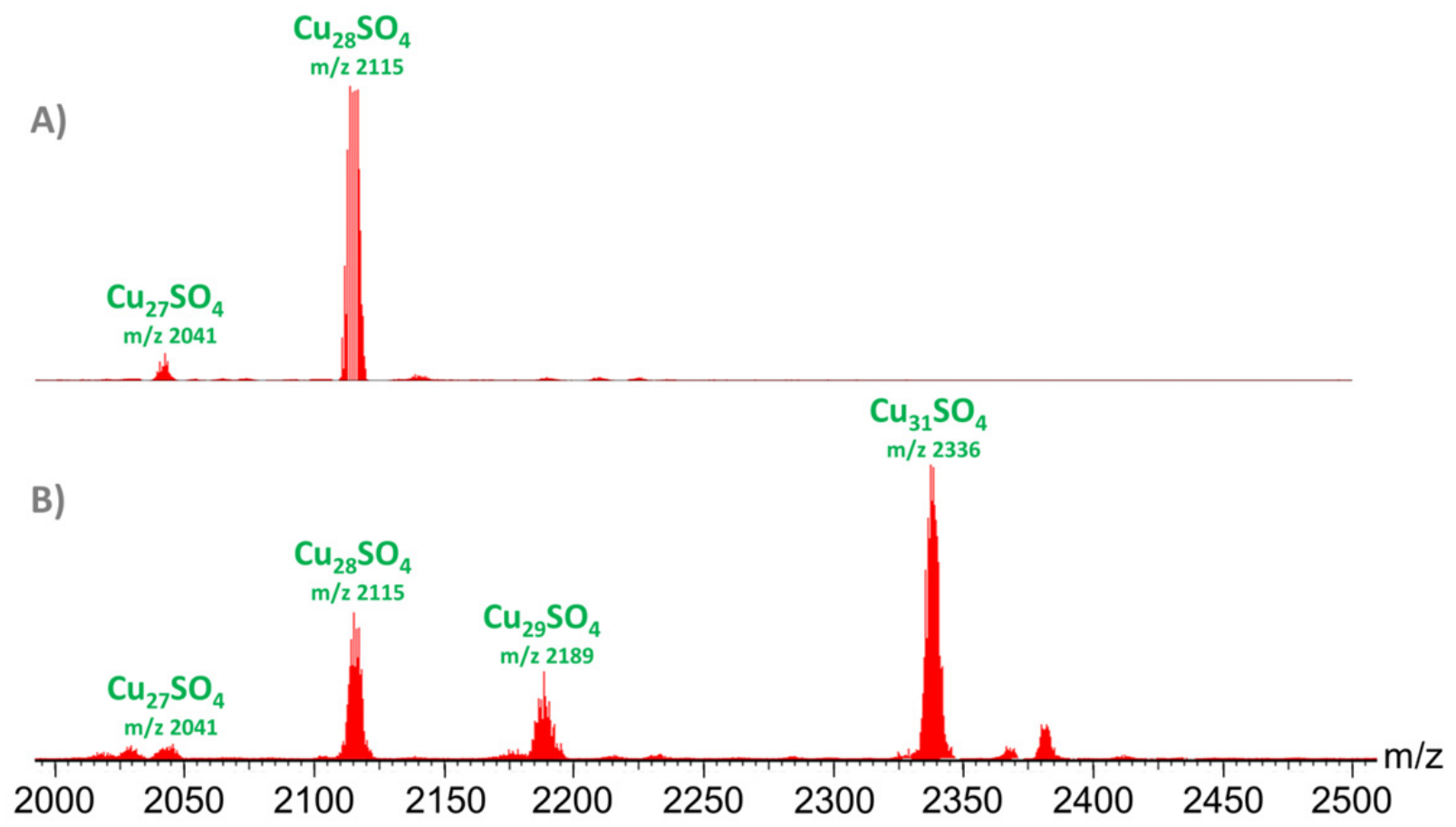

Figure S4. ESI-MS(-) spectra (in $\mathrm{CH}_{3} \mathrm{CN}$ ) of the nanojar fraction containing $\mathrm{Cu}_{28} \mathrm{SO}_{4}$ and small amounts of $\mathrm{Cu}_{27} \mathrm{SO}_{4}$ before heating $(\mathbf{A})$ and after heating to $150{ }^{\circ} \mathrm{C}$ in DMSO- $d_{6}(\mathbf{B})$.

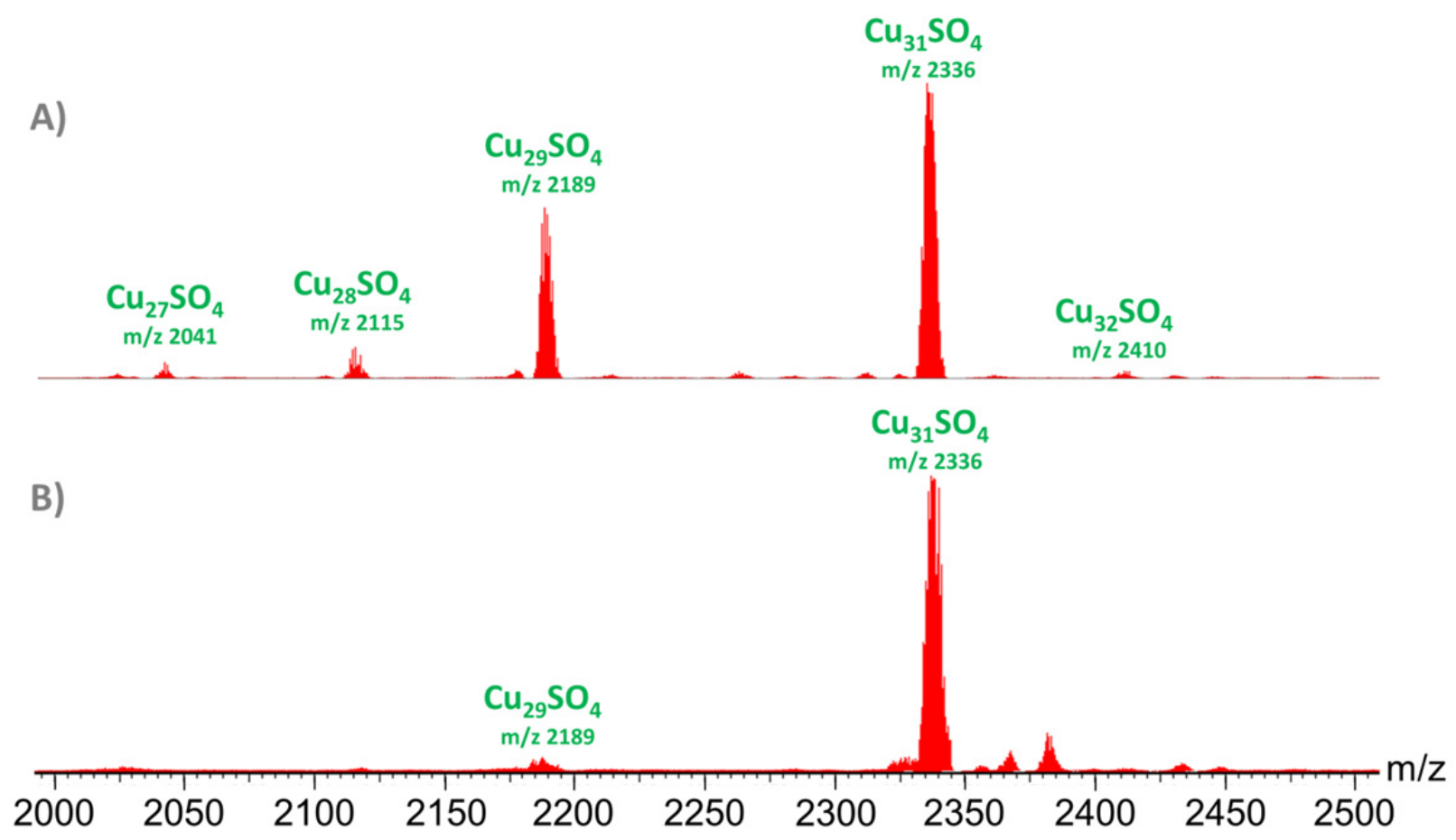

Figure S5. ESI-MS(-) spectra (in $\mathrm{CH}_{3} \mathrm{CN}$ ) of the nanojar fraction containing $\mathrm{Cu}_{29} \mathrm{SO}_{4}$ and $\mathrm{Cu}_{31} \mathrm{SO}_{4}$ (and small amounts of $\mathrm{Cu}_{27} \mathrm{SO}_{4}, \mathrm{Cu}_{28} \mathrm{SO}_{4}$ and $\mathrm{Cu}_{32} \mathrm{SO}_{4}$ ) before heating $(\mathrm{A})$ and after heating to $150{ }^{\circ} \mathrm{C}$ in DMSO- $d_{6}(\mathbf{B})$. 


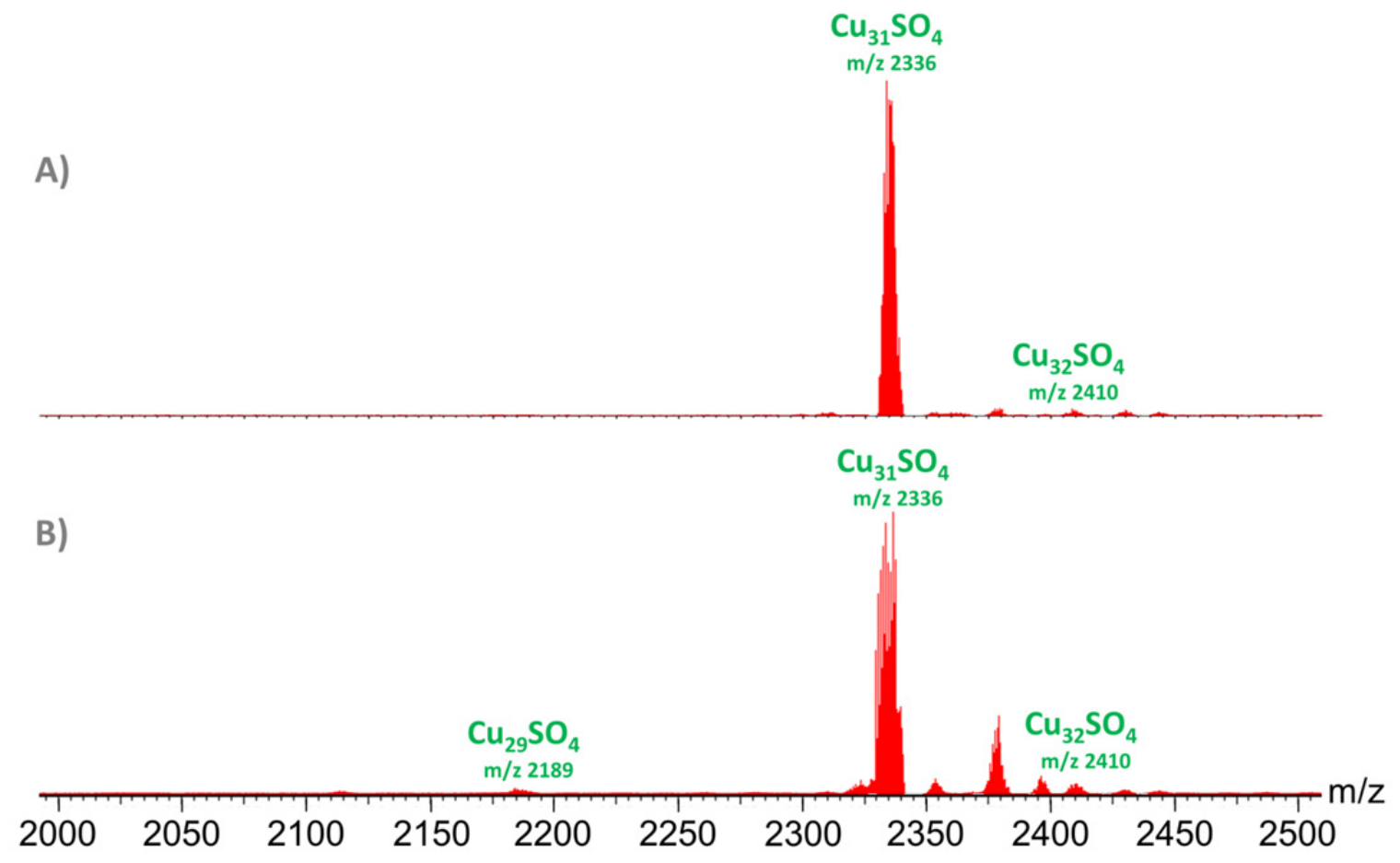

Figure S6. ESI-MS(-) spectra (in $\mathrm{CH}_{3} \mathrm{CN}$ ) of the nanojar fraction containing $\mathrm{Cu}_{31} \mathrm{SO}_{4}$ and small amounts of $\mathrm{Cu}_{32} \mathrm{SO}_{4}$ before heating (A) and after heating to $150{ }^{\circ} \mathrm{C}$ in DMSO- $d_{6}(\mathbf{B})$.

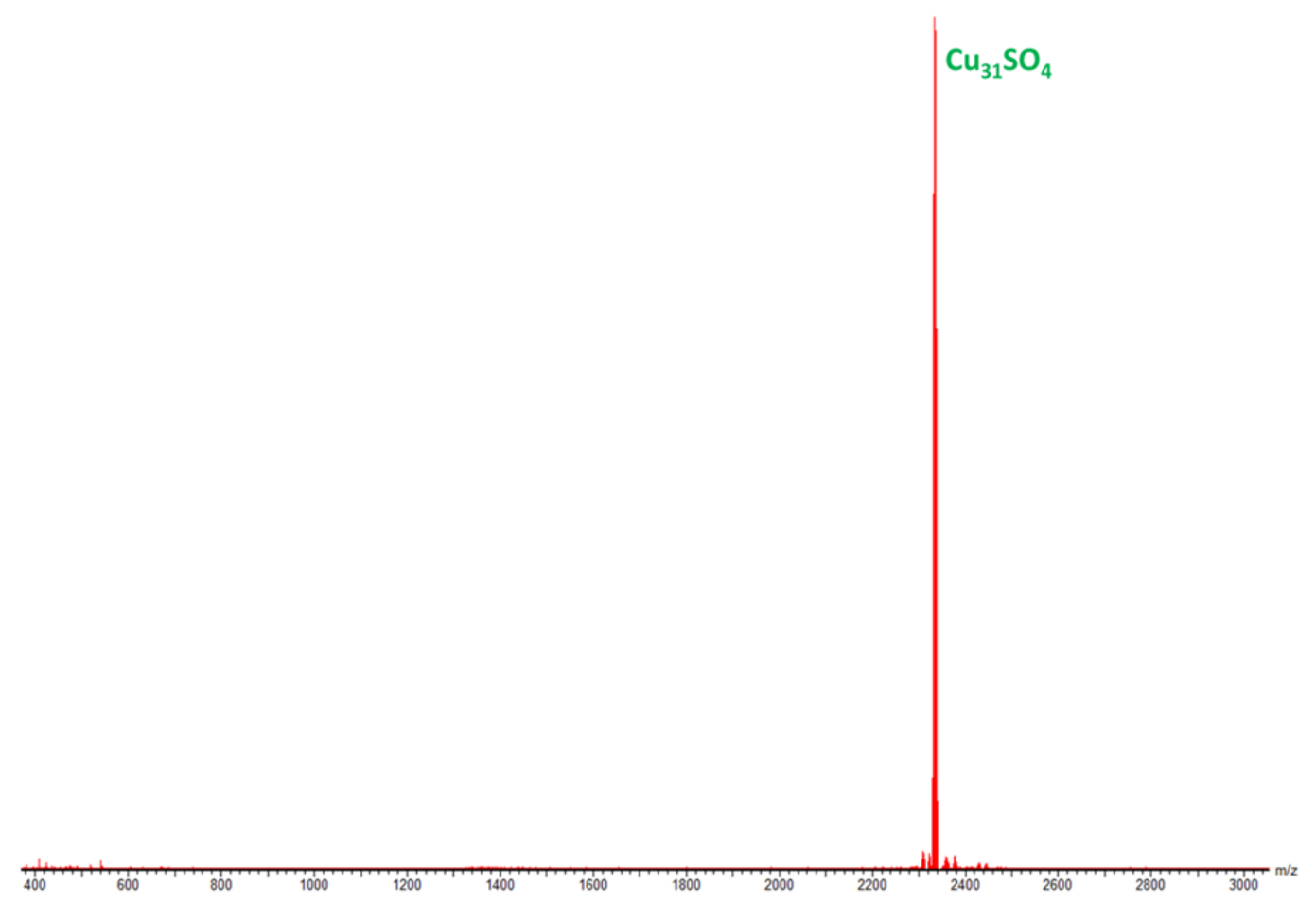

Figure S7. ESI-MS(-) spectrum (in $\left.\mathrm{CH}_{3} \mathrm{CN}\right)$ of $\left(\mathrm{Bu}_{4} \mathrm{~N}\right)_{2}\left[\mathrm{SO}_{4} \subset\{\mathrm{Cu}(\mathrm{OH})(\mathrm{pz})\}_{31}\right]$. Minor peaks at $\mathrm{m} / z$ 2312.8, 2323.9, 2355.9 and 2376.4 correspond to adducts formed during ionization, $\left[\mathrm{SO}_{4} \subset\left\{\mathrm{Cu}_{31}(\mathrm{OH})_{31}(\mathrm{pz})\right\}_{31-\mathrm{x}}(\mathrm{HCOO})_{\mathrm{x}}\right]^{2-}$ and $\left[\mathrm{SO}_{4} \subset\left\{\mathrm{Cu}_{31}(\mathrm{OH})_{31}(\mathrm{pz})\right\}_{30}\left(\mathrm{CH}_{3} \mathrm{CN}\right)_{\mathrm{x}}\right]^{2-}(\mathrm{x}=1,2)$, respectively. 


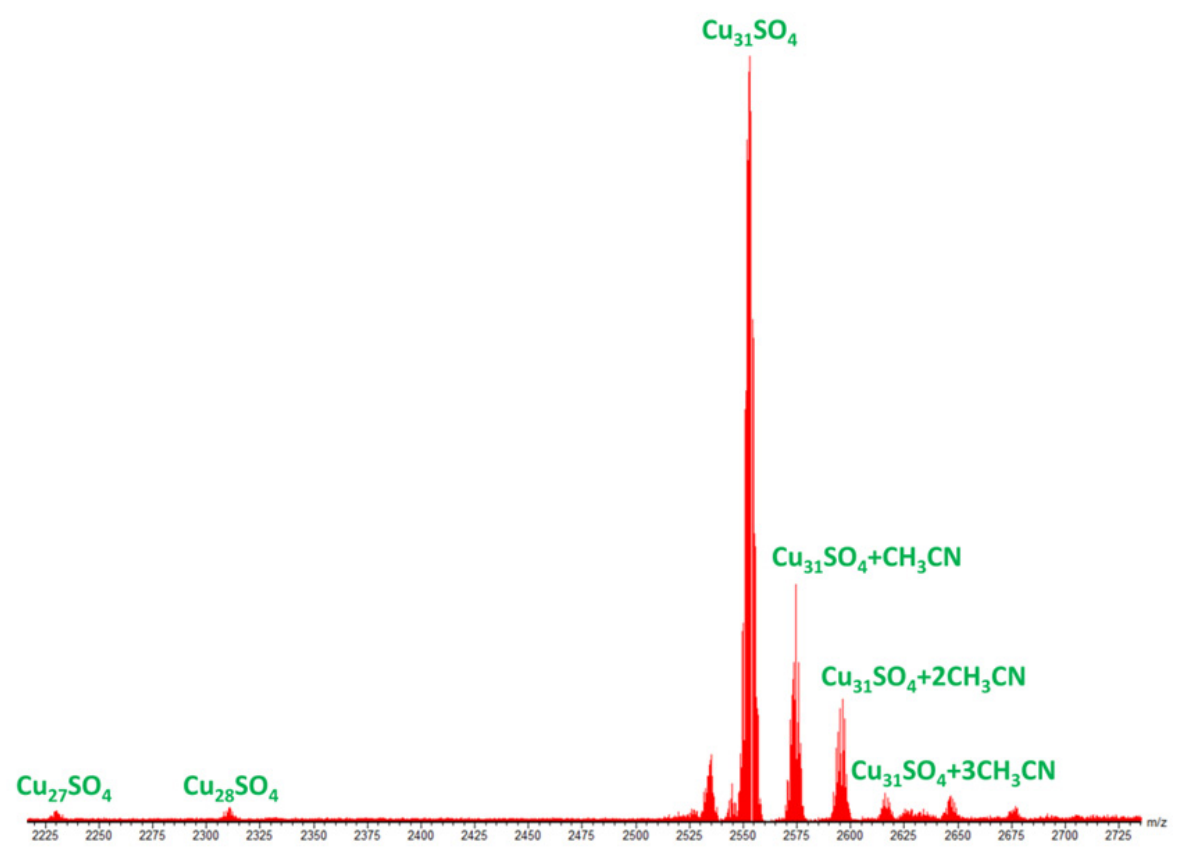

Figure S8. ESI-MS(-) spectrum (in $\left.\mathrm{CH}_{3} \mathrm{CN}\right)$ of $\left(\mathrm{Bu}_{4} \mathrm{~N}\right)_{2}\left[\mathrm{SO}_{4} \subset\{\mathrm{Cu}(\mathrm{OH})(4-\mathrm{Mepz})\}_{31}\right]$. The peak at $m / z$ 2535 corresponds to $\left[\mathrm{SO}_{4} \subset\left\{\mathrm{Cu}_{31}(\mathrm{OH})_{31}(4-\mathrm{Mepz})\right\}_{30}(\mathrm{HCOO})\right]^{2-}$.
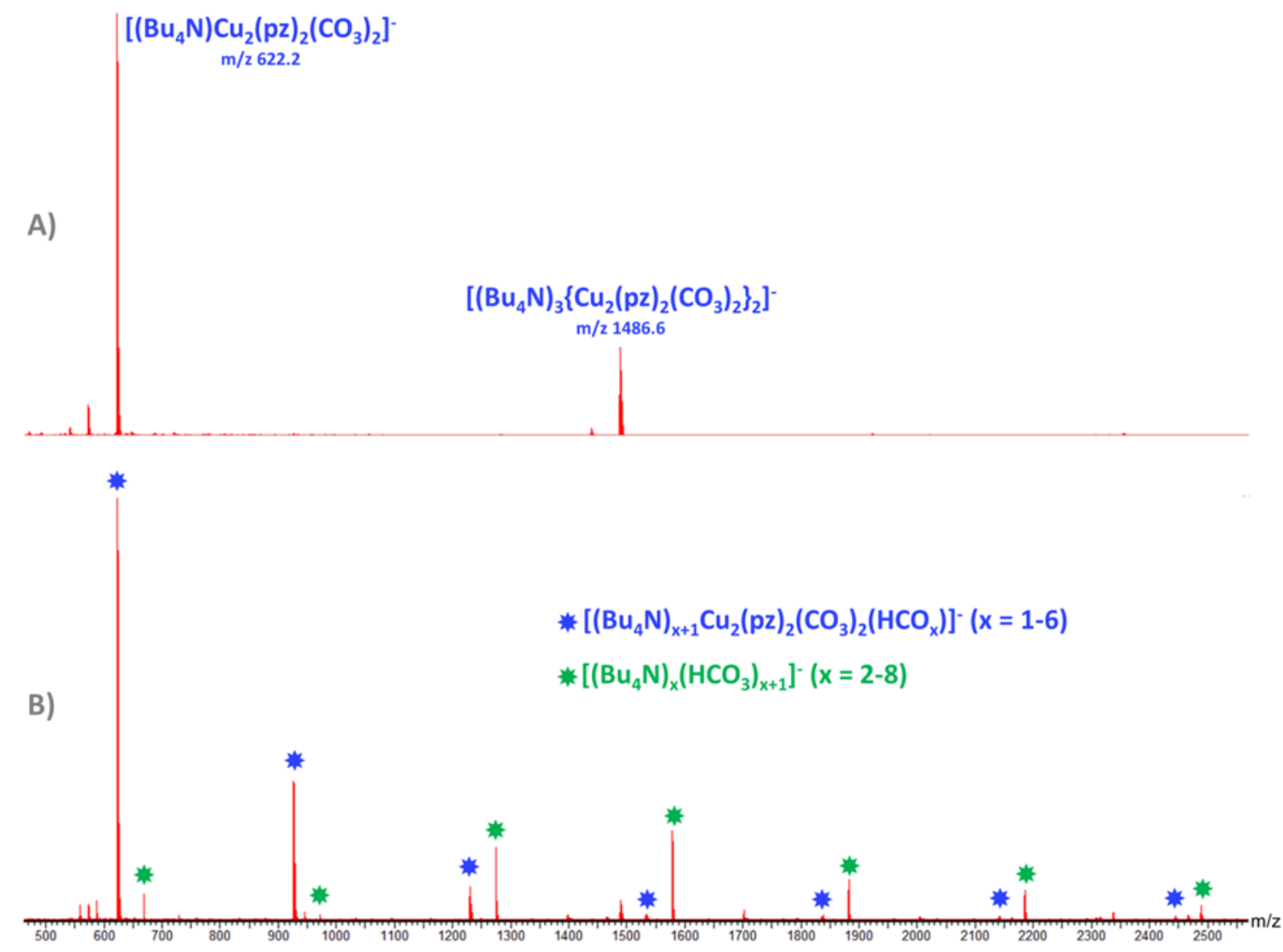

Figure S9. ESI-MS(-) spectrum (in $\left.\mathrm{CH}_{3} \mathrm{CN}\right)$ of isolated $\left(\mathrm{Bu}_{4} \mathrm{~N}\right)_{2}\left[\mathrm{Cu}_{2}(\mathrm{pz})_{2}\left(\mathrm{CO}_{3}\right)_{2}\right](\mathbf{A})$, and its mother liquor $(\mathbf{B})$. The peaks at $m / z 572.2$ and 1438.6 correspond to $\left[\left(\mathrm{Bu}_{4} \mathrm{~N}\right) \mathrm{Cu}_{2}(\mathrm{OH})(\mathrm{pz})\left(\mathrm{CO}_{3}\right)_{2}\right]^{-}$and $\left[\left(\mathrm{Bu}_{4} \mathrm{~N}\right)_{3} \mathrm{Cu}_{4}(\mathrm{OH})(\mathrm{pz})_{3}\left(\mathrm{CO}_{3}\right)_{4}\right]^{-}$, respectively. 
A)

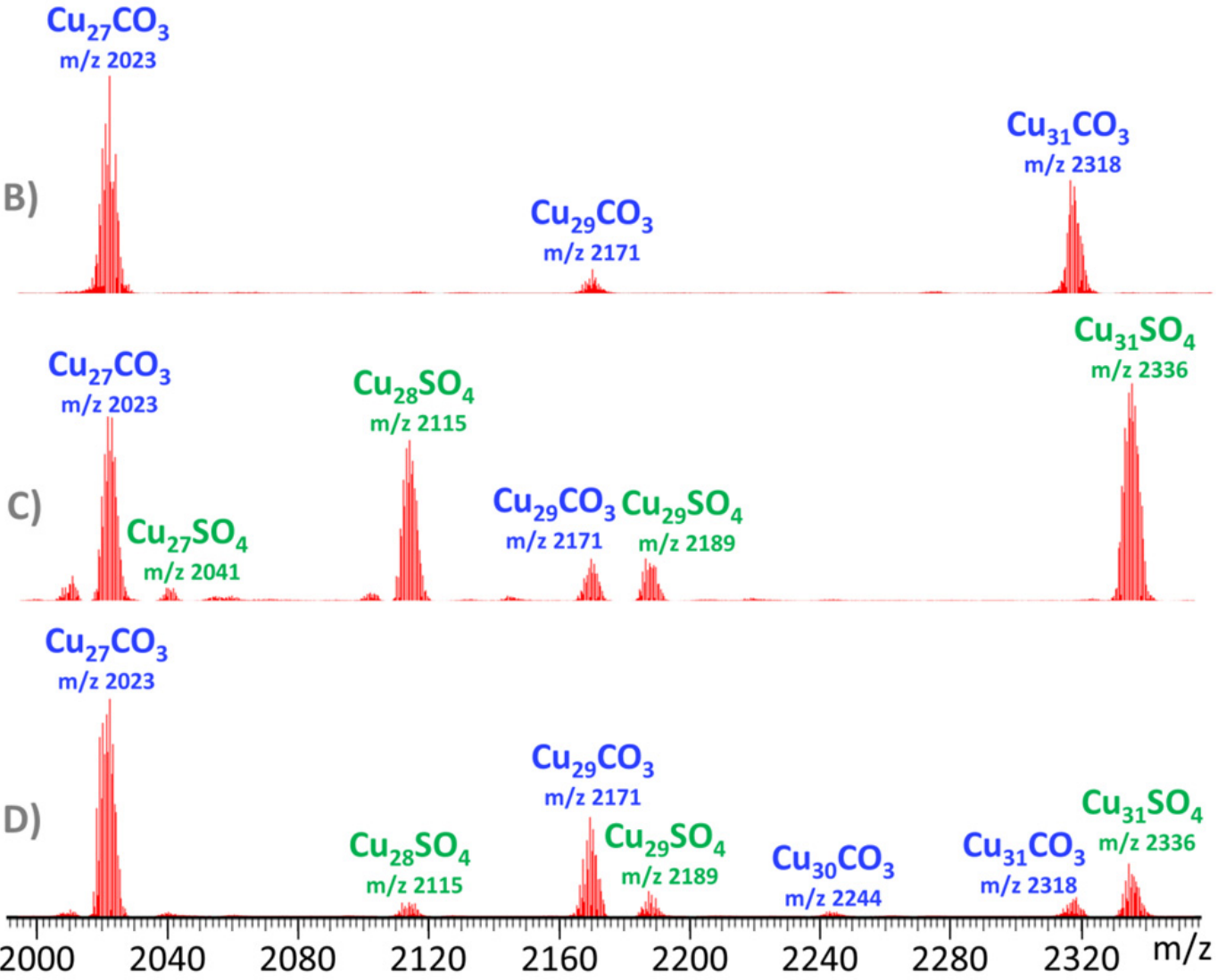

Figure S10. ESI-MS(-) spectra (in $\mathrm{CH}_{3} \mathrm{CN}$ ) of the reaction product in THF of $\mathrm{Cu}\left(\mathrm{NO}_{3}\right)_{2}, \mathrm{CuSO}_{4}$, pyrazole, $\mathrm{NaOH}, \mathrm{Na}_{2} \mathrm{CO}_{3}$ and $\mathrm{Bu}_{4} \mathrm{NOH}(29: 1: 30: 56: 1: 2)(\mathbf{A}), \mathrm{Cu}\left(\mathrm{NO}_{3}\right)_{2}, \mathrm{Na}_{2} \mathrm{SO}_{4}$, pyrazole, $\mathrm{NaOH}$,

$\mathrm{Na}_{2} \mathrm{CO}_{3}$ and $\mathrm{Bu}_{4} \mathrm{NOH}(29: 29: 29: 56: 29: 2)(\mathbf{B}), \mathrm{CuSO}_{4}$, pyrazole, $\mathrm{NaOH}, \mathrm{Na}_{2} \mathrm{CO}_{3}$ and $\mathrm{Bu}_{4} \mathrm{NOH}$ (29:29:56:29:2) (C), and $\mathrm{CuCO}_{3} \cdot \mathrm{Cu}(\mathrm{OH})_{2}, \mathrm{PbSO}_{4}$, pyrazole, $\mathrm{NaOH}$ and $\mathrm{Bu}_{4} \mathrm{NOH}(29: 29: 58: 54: 4)(\mathbf{D})$.

Molar ratios are indicated in parentheses. The $\mathrm{SO}_{4}{ }^{2-}: \mathrm{CO}_{3}{ }^{2-}$ molar ratio is 1:1 in all reactions. 


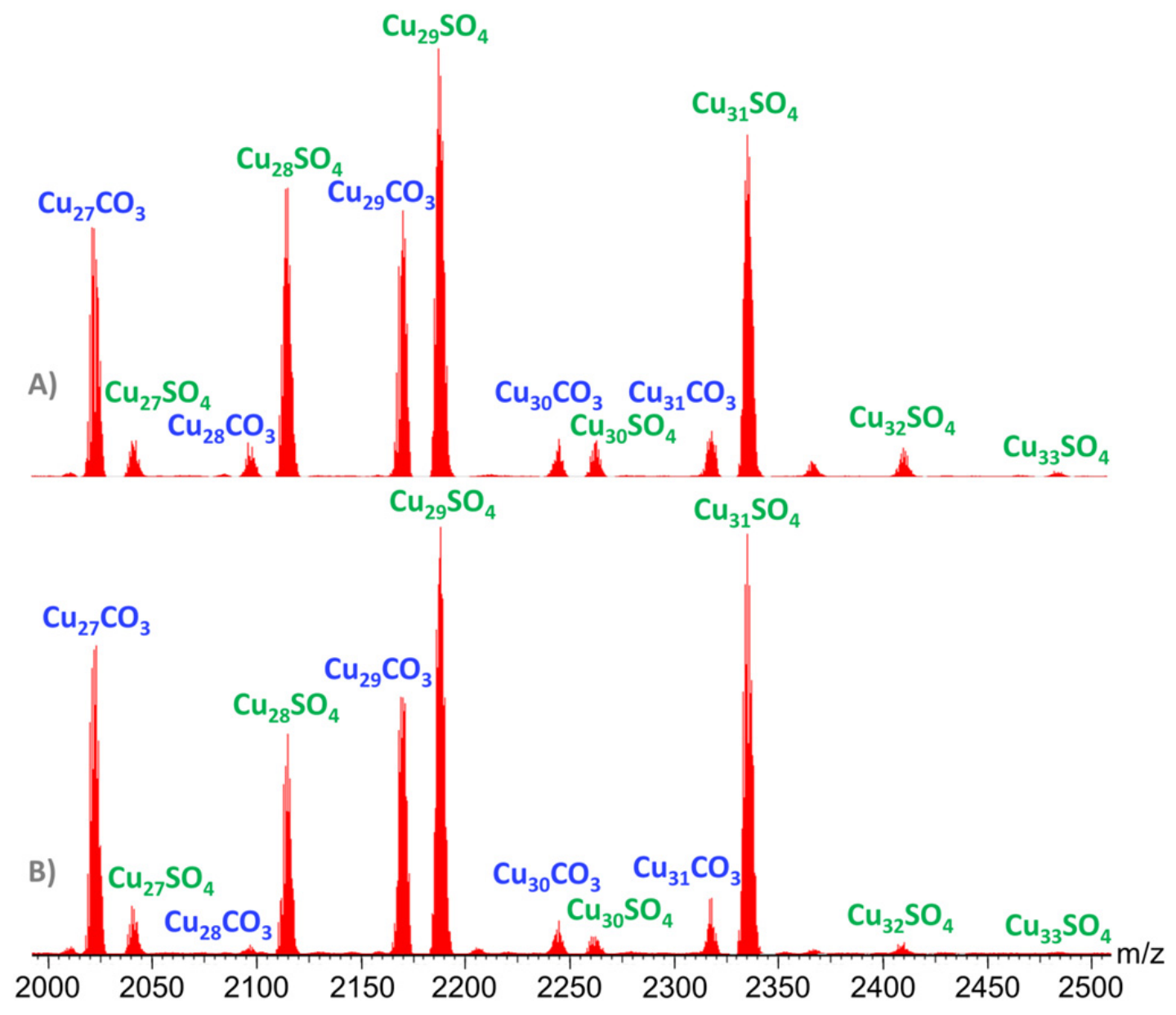

Figure S11. ESI-MS(-) spectra (in $\mathrm{CH}_{3} \mathrm{CN}$ ) of a mixture of sulfate- and carbonate-incarcerating nanojars obtained at room temperature with a $\mathrm{SO}_{4}{ }^{2-}: \mathrm{CO}_{3}{ }^{2-}$ molar ratio of $1: 1$, when all reagents are soluble in the THF reaction medium (A), and after heating the solid mixture for 24 hours at $140{ }^{\circ} \mathrm{C}$ under air (B). 


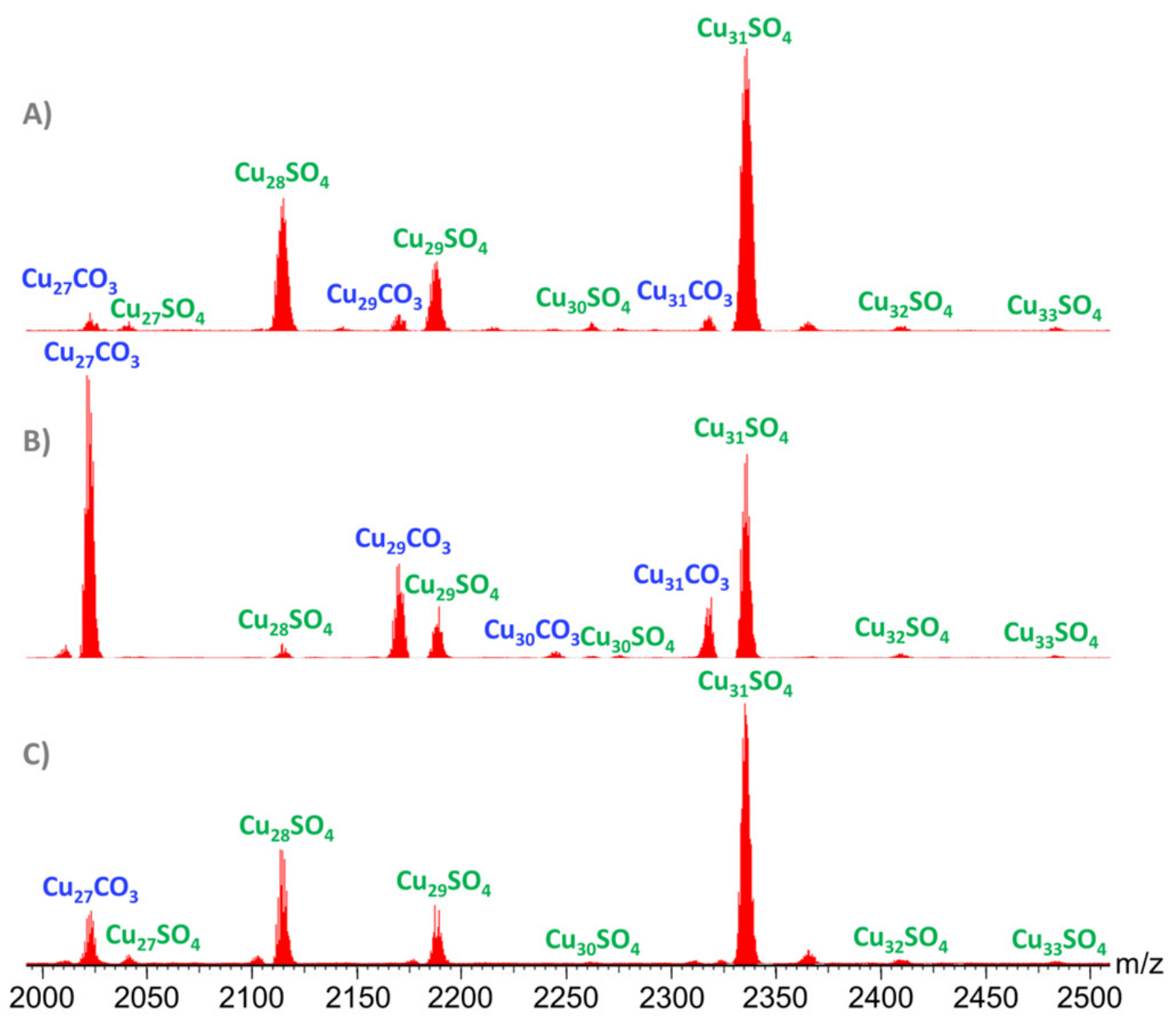

Figure S12. ESI-MS(-) spectra (in $\mathrm{CH}_{3} \mathrm{CN}$ ) demonstrating extraction of sulfate by nanojars from water into $\mathrm{CH}_{2} \mathrm{Cl}_{2}$, using $\mathrm{CuSO}_{4}$, pyrazole, $\mathrm{KOH}$ and $\mathrm{Bu}_{4} \mathrm{NOH}$ in a 28:28:56:2 molar ratio (A), into THF, using $\mathrm{Cu}\left(\mathrm{NO}_{3}\right)_{2}$, pyrazole, $\mathrm{Bu}_{4} \mathrm{NOH},\left(\mathrm{Bu}_{4} \mathrm{~N}\right)_{2} \mathrm{SO}_{4}$ and $\left(\mathrm{Bu}_{4} \mathrm{~N}\right)_{2} \mathrm{CO}_{3}$ in a 29:29:58:1:1 molar ratio $(\mathbf{B})$, and into THF, using $\mathrm{Cu}\left(\mathrm{NO}_{3}\right)_{2}$, pyrazole, $\mathrm{NaOH}, \mathrm{Bu}_{4} \mathrm{NOH}, \mathrm{Na}_{2} \mathrm{SO}_{4}$ and $\mathrm{Na}_{2} \mathrm{CO}_{3}$ in a 29:29:56:2:125:1 molar ratio (C). 
Table S1. Variable-temperature ${ }^{1} \mathrm{H}$ NMR chemical shifts (ppm) of nanojars $\mathbf{C u}_{\mathrm{n}} \mathbf{S O}_{4}(\mathrm{n}=27-32)$ in DMSO- $d_{6}$.

\begin{tabular}{|c|c|c|c|c|c|c|c|c|c|c|c|c|c|c|}
\hline Nanojar & $25^{\circ} \mathrm{C}$ & $30^{\circ} \mathrm{C}$ & $40^{\circ} \mathrm{C}$ & $50{ }^{\circ} \mathrm{C}$ & $60^{\circ} \mathrm{C}$ & $70^{\circ} \mathrm{C}$ & $80^{\circ} \mathrm{C}$ & $90^{\circ} \mathrm{C}$ & $100^{\circ} \mathrm{C}$ & $110^{\circ} \mathrm{C}$ & $120{ }^{\circ} \mathrm{C}$ & $130{ }^{\circ} \mathrm{C}$ & $140{ }^{\circ} \mathrm{C}$ & $150{ }^{\circ} \mathrm{C}$ \\
\hline \multicolumn{15}{|l|}{$\mathrm{Cu}_{27}(6+12+9)$} \\
\hline $\mathrm{Cu}_{9}$-ring, pz-4- $H$ & 34.83 & 34.66 & 34.31 & 33.93 & 33.55 & 33.17 & 32.77 & 32.39 & 32.01 & 31.64 & 31.29 & 30.94 & 30.63 & 30.28 \\
\hline Cug-ring, pz-3,5- $\mathrm{H}_{2}$ & 31.03 & 30.89 & 30.62 & 30.31 & 30.01 & 29.69 & 29.37 & 29.05 & 28.73 & 28.42 & 28.11 & 27.82 & 27.52 & 27.24 \\
\hline $\mathrm{Cu}_{6}$-ring, pz-4-H & $\sim 33.70$ & $\sim 33.65$ & 33.58 & 33.50 & 33.40 & 33.27 & 33.15 & 33.00 & 32.85 & 32.69 & 32.53 & 32.36 & 32.18 & 31.98 \\
\hline $\mathrm{Cu}_{6}$-ring, pz-3,5- $\mathrm{H}_{2}$ & 30.18 & 30.18 & 30.15 & 30.08 & 30.01 & 29.92 & 29.82 & 29.70 & 29.58 & 29.46 & 29.32 & 29.17 & 29.02 & 28.86 \\
\hline $\mathrm{Cu}_{12}$-ring, pz-4-H(9) & 28.04 & 28.07 & 28.13 & 28.19 & 28.22 & 28.25 & 28.27 & 28.27 & 28.27 & 28.25 & 28.22 & 28.19 & 28.15 & 28.10 \\
\hline $\mathrm{Cu}_{12}$-ring, pz-4-H(6) & 26.44 & 26.46 & 26.51 & 26.56 & 26.59 & 26.60 & 26.60 & 26.60 & 26.59 & 26.56 & 26.53 & 26.48 & 26.44 & 26.40 \\
\hline $\mathrm{Cu}_{12}$-ring, pz-3,5- $\mathrm{H}_{2}(9)$ & 22.56 & 22.57 & 22.62 & 22.63 & 22.65 & 22.66 & 22.66 & 22.65 & 22.63 & 22.62 & 22.59 & 22.55 & 22.51 & 22.45 \\
\hline $\mathrm{Cu}_{12}$-ring, pz-3,5- $\mathrm{H}_{2}(6)$ & 22.56 & 22.57 & 22.62 & 22.63 & 22.65 & 22.63 & 22.63 & 22.62 & 22.60 & 22.57 & 22.54 & 22.50 & 22.45 & 22.40 \\
\hline $\mathrm{Cu}_{12}$-ring, $\mathrm{OH}(6)$ & -26.18 & -26.24 & -26.33 & -26.47 & -26.57 & -26.62 & -26.68 & -26.68 & -26.65 & -26.66 & -26.51 & -26.50 & -26.50 & -26.37 \\
\hline $\mathrm{Cu}_{12}$-ring, $\mathrm{OH}(9)$ & -42.02 & -42.05 & -41.99 & -41.93 & -41.85 & -41.75 & -41.72 & -41.46 & -41.24 & -41.08 & -40.82 & -40.74 & -40.34 & -40.11 \\
\hline $\mathrm{Cu}_{6}$-ring, $\mathrm{OH}$ & -44.39 & -44.40 & -44.38 & -44.28 & $\sim-44.2$ & $\sim-44.0$ & $\sim-43.8$ & $\sim-43.7$ & $\sim-43.6$ & $\sim-43.4$ & -42.98 & -42.72 & -42.45 & -42.21 \\
\hline $\mathrm{Cu}_{9}$-ring, $\mathrm{OH}$ & -52.95 & -52.97 & -52.91 & -52.63 & $\sim-52.3$ & $\sim-52.0$ & $\sim-51.8$ & $\sim-51.2$ & -50.63 & -50.14 & -49.64 & -49.11 & -48.63 & -48.11 \\
\hline \multicolumn{15}{|l|}{$\mathrm{Cu}_{28}(6+12+10)$} \\
\hline $\mathrm{Cu}_{10}$-ring, pz-4- $H$ & 40.27 & 39.99 & 39.46 & 38.94 & 38.42 & 37.92 & 37.43 & 36.94 & 36.47 & 36.01 & 35.56 & 35.12 & 34.69 & 34.28 \\
\hline $\mathrm{Cu}_{10}$-ring, pz-3,5- $\mathrm{H}_{2}$ & 34.50 & 34.27 & 33.81 & 33.37 & 32.94 & 32.51 & 32.08 & 31.67 & 31.27 & 30.89 & 30.51 & 30.15 & 29.81 & 29.41 \\
\hline $\mathrm{Cu}_{6}$-ring, pz-4- $H$ & 33.61 & 33.63 & 33.67 & 33.67 & 33.64 & 33.60 & 33.52 & 33.41 & 33.31 & 33.17 & 33.03 & 32.86 & 32.70 & 32.54 \\
\hline $\mathrm{Cu}_{6}$-ring, pz-3,5- $\mathrm{H}_{2}$ & 30.77 & 30.79 & 30.80 & 30.79 & 30.74 & 30.68 & 30.59 & 30.48 & 30.37 & 30.24 & 30.10 & 29.92 & 29.61 & 29.41 \\
\hline $\mathrm{Cu}_{12}$-ring, pz-4-H(10) & 28.97 & 29.00 & 29.06 & 29.09 & 29.12 & 29.14 & 29.14 & 29.12 & 29.11 & 29.08 & 29.03 & 28.98 & 28.92 & 28.86 \\
\hline $\mathrm{Cu}_{12}$-ring, pz-4-H(6) & 27.48 & 27.50 & 27.56 & 27.60 & 27.61 & 27.63 & 27.61 & 27.60 & 27.56 & 27.52 & 27.47 & 27.41 & 27.35 & $\sim 27.3$ \\
\hline $\mathrm{Cu}_{12}$-ring, pz-3,5- $\mathrm{H}_{2}(10)$ & 23.40 & 23.41 & 23.43 & 23.44 & 23.46 & 23.44 & 23.43 & 23.40 & 23.37 & 23.32 & 23.27 & 23.23 & 23.17 & 23.09 \\
\hline $\mathrm{Cu}_{12}$-ring, pz-3,5- $\mathrm{H}_{2}(6)$ & 23.23 & 23.24 & 23.27 & 23.29 & 23.29 & 23.29 & 23.27 & 23.24 & 23.23 & 23.18 & 23.14 & 23.09 & 23.05 & 22.98 \\
\hline $\mathrm{Cu}_{12}$-ring, $\mathrm{OH}(6)$ & -32.98 & -32.91 & -32.74 & -32.57 & -32.42 & -32.19 & -32.01 & -31.79 & -31.53 & -31.32 & -31.06 & -30.79 & -30.53 & $\sim-30.2$ \\
\hline $\mathrm{Cu}_{12}$-ring, $\mathrm{OH}(10)$ & -43.47 & -43.56 & -43.69 & -43.78 & -43.81 & -43.84 & -43.85 & -43.76 & -43.61 & -43.41 & $\sim-43.2$ & $\sim-43.1$ & $\sim-43.0$ & $\sim-42.9$ \\
\hline $\mathrm{Cu}_{6}$-ring, $\mathrm{OH}$ & -51.49 & -51.66 & -51.95 & -52.13 & -52.27 & -52.16 & $\sim-52.0$ & -52.07 & -51.88 & -51.66 & -51.35 & -51.08 & -50.69 & -50.27 \\
\hline $\mathrm{Cu}_{10}$-ring, $\mathrm{OH}$ & -57.75 & -57.18 & -56.10 & -55.05 & -53.95 & -52.94 & $\sim-51.9$ & $\sim-51.0$ & -50.02 & -49.09 & -48.19 & -47.29 & -46.48 & -45.67 \\
\hline \multicolumn{15}{|l|}{$\mathrm{Cu}_{29}(8+13+8)$} \\
\hline $\mathrm{Cu}_{8}$-ring, pz-4- $H$ & 28.33 & 28.31 & 28.30 & 28.28 & 28.25 & 28.22 & 28.19 & 28.14 & 28.11 & 28.05 & 28.01 & 27.95 & 27.89 & 27.82 \\
\hline Cus-ring, pz-3,5- $\mathrm{H}_{2}$ & 25.63 & 25.63 & 25.61 & 25.60 & 25.56 & 25.55 & 25.52 & 25.47 & 25.43 & 25.38 & 25.34 & 25.27 & 25.23 & 25.15 \\
\hline $\mathrm{Cu}_{13}$-ring, pz-4- $H$ & 26.59 & 26.65 & 26.74 & 26.82 & 26.88 & 26.94 & 26.98 & 27.02 & 27.03 & 27.05 & 27.06 & 27.06 & 27.05 & 27.03 \\
\hline $\mathrm{Cu}_{13}$-ring, $\mathrm{pz}-3,5-\mathrm{H}_{2}$ & 21.67 & 21.70 & 21.75 & 21.79 & 21.84 & 21.85 & 21.87 & 21.89 & 21.89 & 21.89 & 21.87 & 21.85 & 21.82 & 21.81 \\
\hline $\mathrm{Cu}_{13}$-ring, $\mathrm{OH}$ & -28.57 & -28.65 & -28.86 & -29.08 & -29.18 & -29.34 & -29.49 & -29.60 & -29.69 & -29.76 & -29.89 & -29.93 & -29.99 & -30.07 \\
\hline $\mathrm{Cu}_{8}$-ring, $\mathrm{OH}$ & -38.88 & -38.57 & -38.37 & -38.01 & -37.76 & -37.56 & -37.31 & -37.11 & -36.86 & -36.73 & -36.52 & -36.37 & -36.04 & -35.11 \\
\hline \multicolumn{15}{|l|}{$\mathrm{Cu}_{29}(7+13+9)$} \\
\hline Cu9-ring, pz-4- $H$ & 31.84 & 31.75 & 31.56 & 31.38 & 31.18 & 30.98 & 30.78 & 30.59 & 30.40 & 30.21 & $\sim 30.00$ & $\sim 29.85$ & $\sim 29.67$ & 29.49 \\
\hline $\mathrm{Cu}_{9}$-ring, pz-3,5- $\mathrm{H}_{2}$ & 28.63 & 28.56 & 28.39 & 28.21 & 28.04 & 27.85 & 27.67 & 27.49 & 27.31 & 27.13 & 26.95 & 26.78 & $\sim 26.59$ & $\sim 26.45$ \\
\hline $\mathrm{Cu}_{7}$-ring, pz-4-H & 31.55 & 31.53 & 31.50 & 31.47 & 31.41 & 31.35 & 31.29 & 31.21 & 31.12 & 31.03 & $\sim 30.94$ & $\sim 30.86$ & $\sim 30.67$ & $\sim 30.48$ \\
\hline Cu7-ring, pz-3,5- $\mathrm{H}_{2}$ & 28.16 & 28.16 & 28.13 & 28.11 & $\sim 28.08$ & 28.02 & $\sim 27.97$ & $\sim 27.90$ & 27.82 & 27.73 & 27.65 & 27.56 & $\sim 27.48$ & 27.35 \\
\hline $\mathrm{Cu}_{13}$-ring, pz-4- $H$ & 27.79 & 27.82 & 27.87 & 27.92 & 27.95 & 27.96 & 27.98 & 27.98 & 27.96 & 27.95 & 27.93 & 27.90 & 27.87 & $\sim 27.81$ \\
\hline $\mathrm{Cu}_{13}$-ring, pz-3,5- $\mathrm{H}_{2}$ & 22.51 & 22.53 & 22.54 & 22.56 & 22.57 & 22.57 & 22.56 & 22.56 & 22.53 & 22.50 & 22.47 & 22.44 & 22.39 & 22.34 \\
\hline $\mathrm{Cu}_{13}$-ring, $\mathrm{OH}$ & -30.24 & -30.25 & -30.24 & -30.24 & -30.29 & -30.28 & -30.27 & -30.21 & -30.13 & $\sim-30.1$ & $\sim-30.0$ & $\sim-29.9$ & $\sim-29.8$ & $\sim-29.7$ \\
\hline $\mathrm{Cu}_{7-\text {-ring, } \mathrm{OH}}$ & -42.22 & -42.13 & -42.05 & -41.93 & -41.76 & -41.61 & -41.46 & -41.29 & -41.11 & -40.94 & -40.77 & $\sim-40.6$ & $\sim-40.5$ & $\sim-40.3$ \\
\hline $\mathrm{Cu}_{9}$-ring, $\mathrm{OH}$ & -45.81 & -45.53 & -45.04 & -44.57 & -44.07 & -43.60 & -43.12 & -42.65 & -42.20 & -41.76 & -41.35 & $\sim-40.9$ & $\sim-40.5$ & $\sim-40.1$ \\
\hline \multicolumn{15}{|l|}{$\mathrm{Cu}_{31}(8+14+9)$} \\
\hline Cu9-ring, pz-4- $H$ & 32.95 & 32.79 & 32.62 & 32.39 & 32.19 & 31.99 & 31.77 & 31.60 & 31.40 & 31.21 & 31.03 & 30.86 & 30.70 & 30.51 \\
\hline $\mathrm{Cu}_{9}$-ring, pz-3,5- $\mathrm{H}_{2}$ & 28.73 & 28.76 & 28.57 & 28.40 & 28.25 & 28.10 & 27.93 & 27.78 & 27.63 & 27.49 & 27.33 & 27.20 & 27.06 & 26.91 \\
\hline Cus-ring, pz-4- $H$ & 30.16 & 30.18 & 30.19 & 30.19 & 30.19 & 30.16 & 30.12 & 30.08 & 30.04 & 29.99 & 29.93 & 29.85 & 29.79 & 29.72 \\
\hline $\mathrm{Cu}_{8}$-ring, pz-3,5- $\mathrm{H}_{2}$ & 26.83 & 26.86 & 26.88 & 26.88 & 26.88 & 26.86 & 26.83 & 26.78 & 26.74 & 26.69 & 26.63 & 26.57 & 26.51 & 26.44 \\
\hline $\mathrm{Cu}_{14-\text {-ring, } \mathrm{pz}-4-H}$ & 28.73 & 28.76 & 28.80 & 28.85 & 28.88 & 28.89 & 28.91 & 28.89 & 28.88 & 28.86 & 28.83 & 28.78 & 28.73 & 28.67 \\
\hline $\mathrm{Cu}_{14}$-ring, $\mathrm{pz}-3,5-\mathrm{H}_{2}$ & 22.91 & 22.93 & 22.94 & 22.94 & 22.95 & 22.94 & 22.92 & 22.91 & 22.88 & 22.85 & 22.80 & 22.76 & 22.71 & 22.66 \\
\hline $\mathrm{Cu}_{14}$-ring, $\mathrm{OH}$ & -28.28 & -28.02 & -27.93 & -27.95 & -27.81 & -27.76 & -27.72 & -27.63 & -27.53 & -27.53 & -27.33 & -27.23 & -27.09 & -26.97 \\
\hline Cu8-ring, $\mathrm{OH}$ & - & - & -36.50 & -35.92 & -35.72 & -35.67 & -35.61 & -35.53 & -35.47 & -35.47 & -35.27 & -35.20 & -35.06 & -35.00 \\
\hline $\mathrm{Cu}_{9}$-ring, $\mathrm{OH}$ & - & - & -41.73 & -40.19 & -39.47 & -38.89 & -38.36 & -37.85 & -37.37 & -36.92 & -36.53 & -36.16 & -35.81 & -35.41 \\
\hline \multicolumn{15}{|l|}{$\mathrm{Cu}_{32}(8+14+10)$} \\
\hline $\mathrm{Cu}_{10}$-ring, pz-4- $H$ & 39.69 & 39.52 & 39.12 & 38.73 & 38.33 & 37.93 & $\sim 37.5$ & - & - & - & - & - & - & - \\
\hline $\mathrm{Cu}_{10}$-ring, pz-3,5- $\mathrm{H}_{2}$ & 33.85 & 33.70 & 33.35 & 33.02 & 32.65 & - & - & - & - & - & - & - & - & - \\
\hline $\mathrm{Cu}_{8}$-ring, pz-4- $H$ & 35.82 & 35.72 & 35.37 & 35.23 & 34.97 & 34.70 & 34.43 & 34.16 & 33.91 & 33.66 & 33.40 & 33.15 & $\sim 32.92$ & - \\
\hline Cu8-ring, pz-3,5- $\mathrm{H}_{2}$ & 31.41 & 31.32 & 31.11 & 30.92 & 30.71 & 30.48 & 30.27 & $\sim 30.0$ & 29.82 & 29.62 & 29.41 & 29.20 & $\sim 29.0$ & - \\
\hline $\mathrm{Cu}_{14}$-ring, pz-4- $H$ & 29.15 & 29.18 & 29.21 & 29.23 & 29.24 & 29.24 & 29.24 & 29.21 & 29.18 & 29.15 & 29.11 & 29.06 & $\sim 29.0$ & - \\
\hline $\mathrm{Cu}_{14}$-ring, pz-3,5- $\mathrm{H}_{2}$ & 23.41 & 23.43 & 23.44 & 23.45 & 23.45 & 23.44 & 23.43 & 23.40 & 23.37 & 23.34 & 23.29 & 23.24 & 23.20 & 23.14 \\
\hline $\mathrm{Cu}_{8}$-ring, $\mathrm{OH}$ & -36.07 & -35.99 & -35.96 & -35.84 & -35.76 & -35.64 & $\sim-35.5$ & - & - & - & - & - & - & - \\
\hline $\mathrm{Cu}_{10}$-ring, $\mathrm{OH}$ & -42.21 & -41.99 & -41.73 & -41.46 & -41.09 & -40.74 & -40.39 & $\sim-40.0$ & $\sim-39.6$ & $\sim-39.2$ & $\sim-38.8$ & $\sim-38.4$ & $\sim-38.1$ & $\sim-37.7$ \\
\hline
\end{tabular}




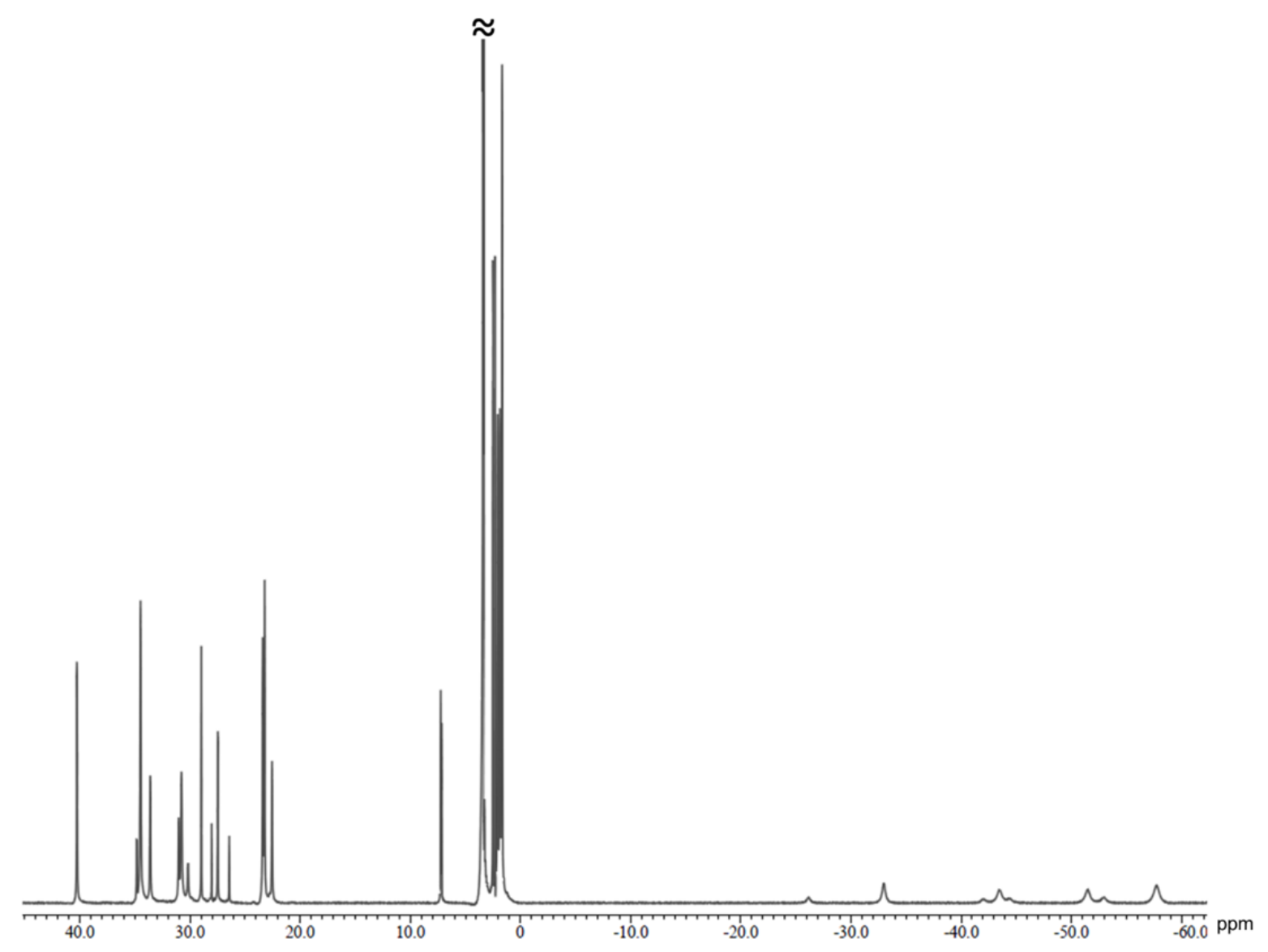

Figure S13. Full ${ }^{1} \mathrm{H}$ NMR spectrum of $\left(\mathrm{Bu}_{4} \mathrm{~N}\right)_{2}\left[\mathrm{SO}_{4} \subset\{\mathrm{Cu}(\mathrm{OH})(\mathrm{pz})\}_{28}\right]$ with small amounts of $\left(\mathrm{Bu}_{4} \mathrm{~N}\right)_{2}\left[\mathrm{SO}_{4} \subset\{\mathrm{Cu}(\mathrm{OH})(\mathrm{pz})\}_{27}\right]$ in DMSO- $d_{6}$ at $25^{\circ} \mathrm{C}$.

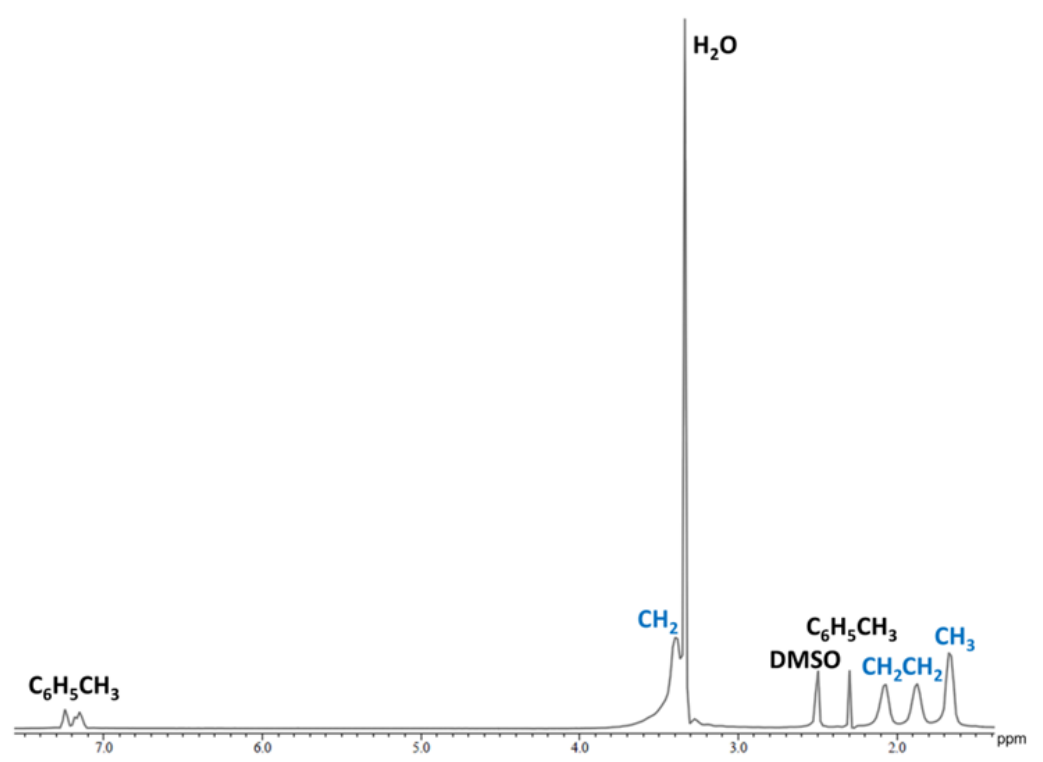

Figure S14. Partial ${ }^{1} \mathrm{H}$ NMR spectrum of $\left(\mathrm{Bu}_{4} \mathrm{~N}\right)_{2}\left[\mathrm{SO}_{4} \subset\{\mathrm{Cu}(\mathrm{OH})(\mathrm{pz})\}_{28}\right]$ with small amounts of $\left(\mathrm{Bu}_{4} \mathrm{~N}\right)_{2}\left[\mathrm{SO}_{4} \subset\{\mathrm{Cu}(\mathrm{OH})(\mathrm{pz})\}_{27}\right]$ in DMSO- $d_{6}$ at $25^{\circ} \mathrm{C}$, showing $\mathrm{Bu}_{4} \mathrm{~N}^{+}$counterion (in blue) and residual solvent peaks. 


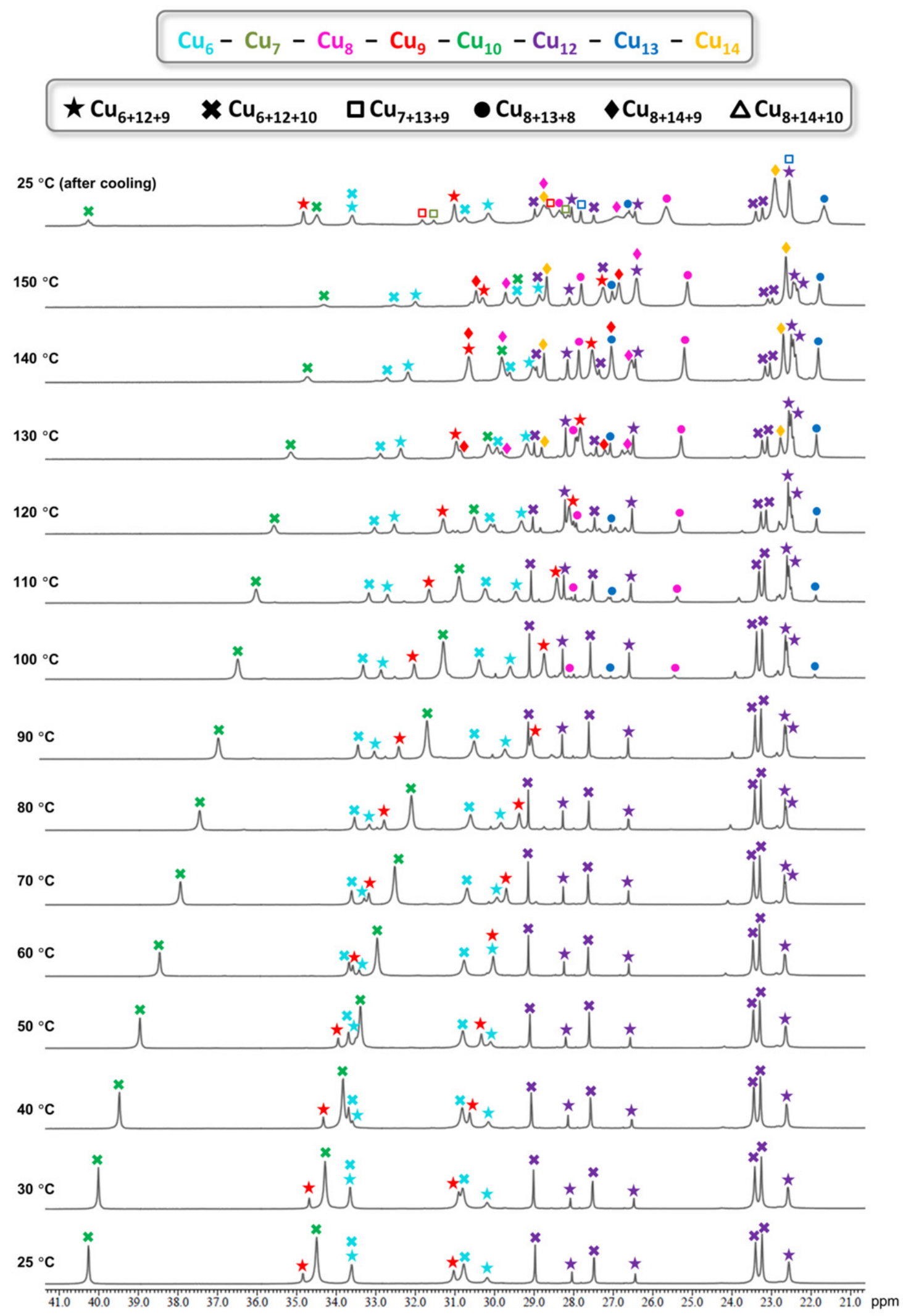

Figure S15. Variable-temperature ${ }^{1} \mathrm{H}$ NMR spectra of $\left(\mathrm{Bu}_{4} \mathrm{~N}\right)_{2}\left[\mathrm{SO}_{4} \subset\{\mathrm{Cu}(\mathrm{OH})(\mathrm{pz})\}_{28}\right]$ with small amounts of $\left(\mathrm{Bu}_{4} \mathrm{~N}\right)_{2}\left[\mathrm{SO}_{4} \subset\{\mathrm{Cu}(\mathrm{OH})(\mathrm{pz})\}_{27}\right]$ in DMSO- $d_{6}$ (pyrazole signals in the 21-41 ppm window). 


$$
\mathrm{Cu}_{6}-\mathrm{Cu}_{7}-\mathrm{Cu}_{8}-\mathrm{Cu}_{9}-\mathrm{Cu}_{10}-\mathrm{Cu}_{12}-\mathrm{Cu}_{13}-\mathrm{Cu}_{14}
$$

\section{$\star \mathrm{Cu}_{6+12+9} \quad \boldsymbol{x} \mathrm{Cu}_{6+12+10} \quad \square \mathrm{Cu}_{7+13+9} \bullet \mathrm{Cu}_{8+13+8} \quad \diamond \mathrm{Cu}_{8+14+9} \quad \Delta \mathrm{Cu}_{8+14+10}$}

$25^{\circ} \mathrm{C}$ (after cooling)

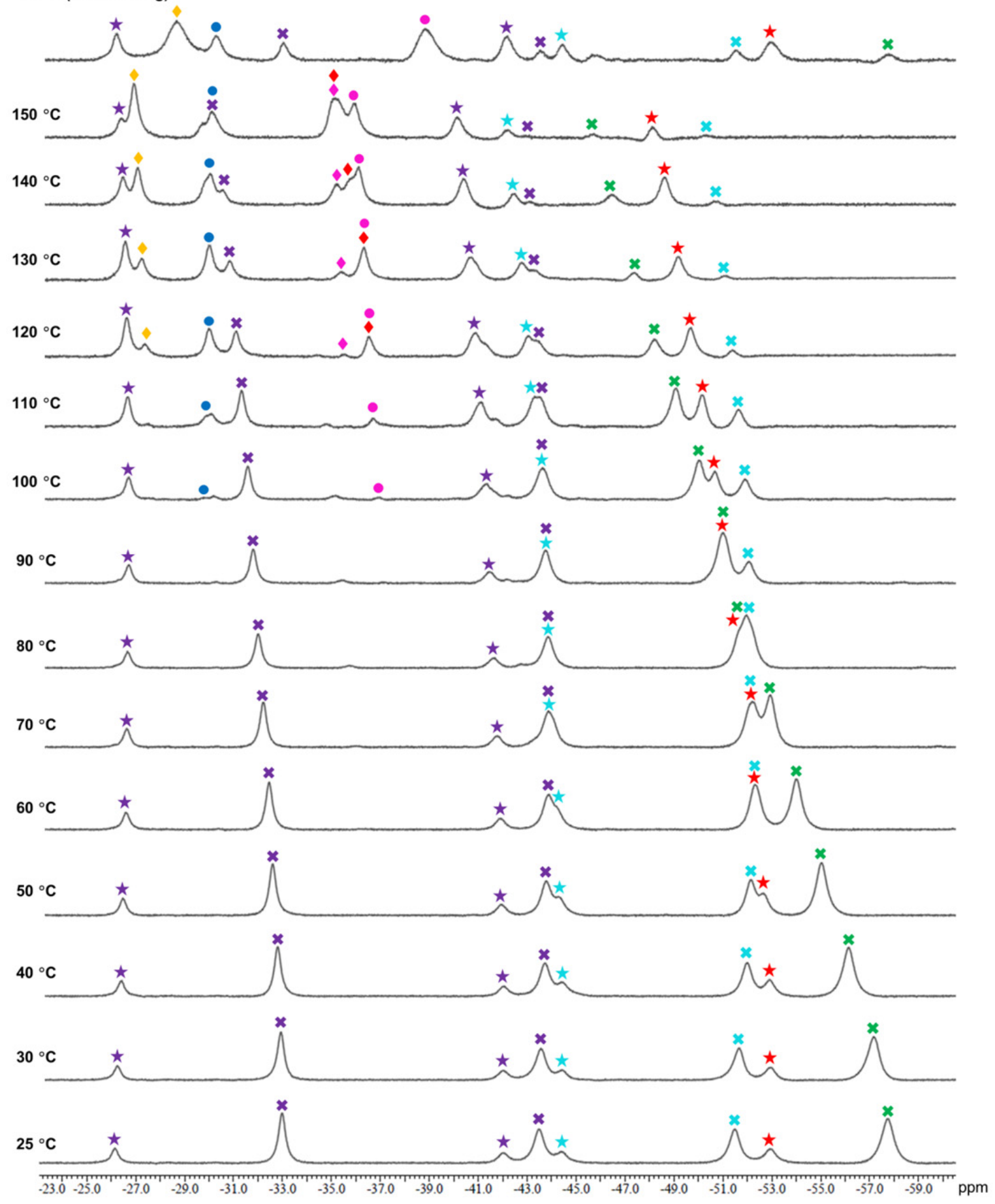

Figure S16. Variable-temperature ${ }^{1} \mathrm{H}$ NMR spectra of $\left(\mathrm{Bu}_{4} \mathrm{~N}\right)_{2}\left[\mathrm{SO}_{4} \subset\{\mathrm{Cu}(\mathrm{OH})(\mathrm{pz})\}_{28}\right]$ with small amounts of $\left(\mathrm{Bu}_{4} \mathrm{~N}\right)_{2}\left[\mathrm{SO}_{4} \subset\{\mathrm{Cu}(\mathrm{OH})(\mathrm{pz})\}_{27}\right]$ in DMSO-d $d_{6}(\mathrm{OH}$ signals in the (-25)-(-59) ppm window). 


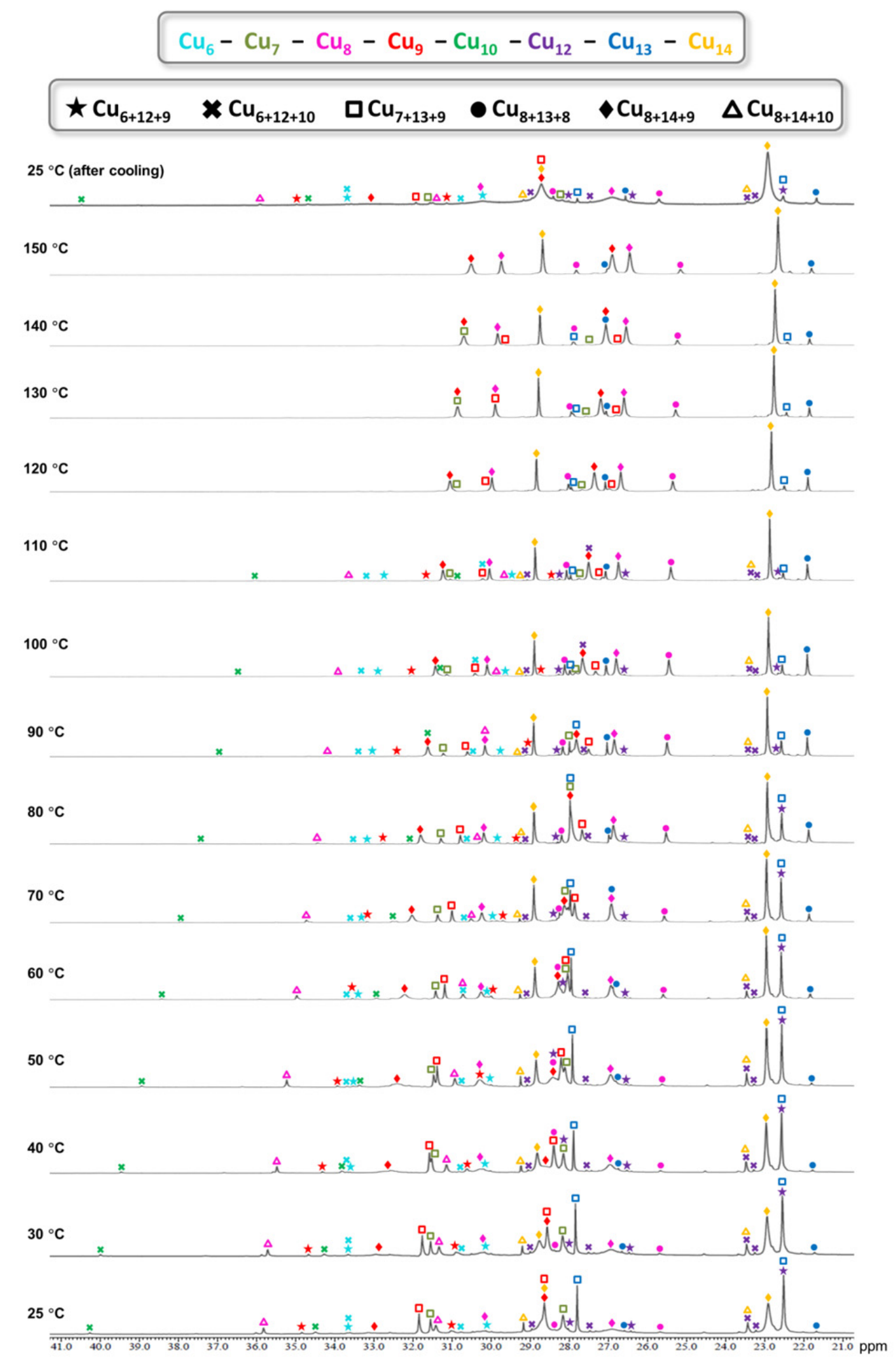

Figure S17. Variable-temperature ${ }^{1} \mathrm{H}$ NMR spectra of $\left(\mathrm{Bu}_{4} \mathrm{~N}\right)_{2}\left[\mathrm{SO}_{4} \subset\{\mathrm{Cu}(\mathrm{OH})(\mathrm{pz})\}_{29}\right]$ and $\left(\mathrm{Bu}_{4} \mathrm{~N}\right)_{2}\left[\mathrm{SO}_{4} \subset\{\mathrm{Cu}(\mathrm{OH})(\mathrm{pz})\}_{31}\right]$, with small amounts of $\mathrm{Cu}_{27} \mathrm{SO}_{4}, \mathrm{Cu}_{28} \mathrm{SO}_{4}$ and $\mathrm{Cu}_{32} \mathrm{SO}_{4}$ in DMSO-d (pyrazole signals in the 21-41 ppm window). 


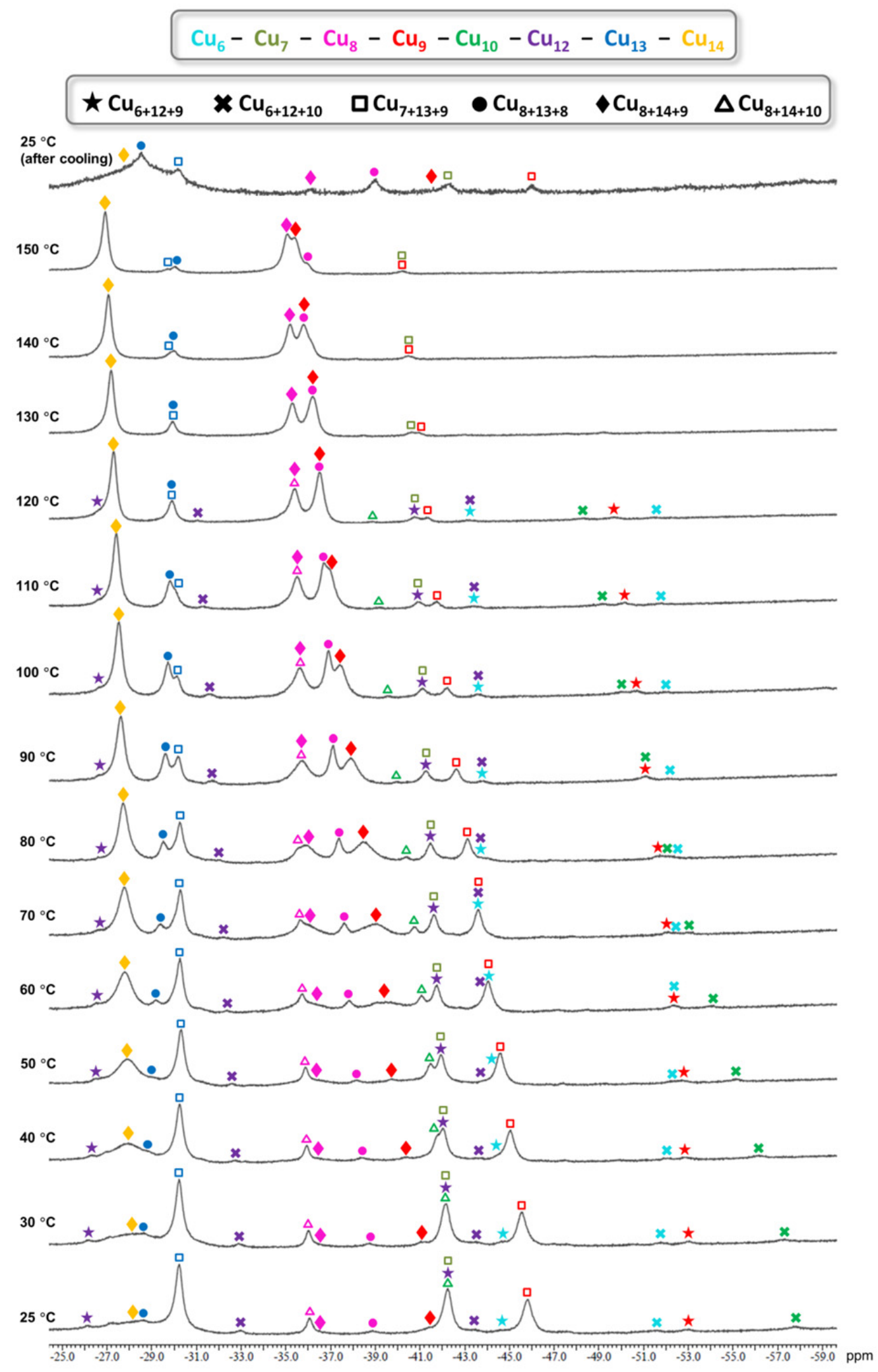

Figure S18. Variable-temperature ${ }^{1} \mathrm{H}$ NMR spectra of $\left(\mathrm{Bu}_{4} \mathrm{~N}\right)_{2}\left[\mathrm{SO}_{4} \subset\{\mathrm{Cu}(\mathrm{OH})(\mathrm{pz})\}_{29}\right]$ and $\left(\mathrm{Bu}_{4} \mathrm{~N}\right)_{2}\left[\mathrm{SO}_{4} \subset\{\mathrm{Cu}(\mathrm{OH})(\mathrm{pz})\}_{31}\right]$, with small amounts of $\mathrm{Cu}_{27} \mathrm{SO}_{4}, \mathrm{Cu}_{28} \mathrm{SO}_{4}$ and $\mathrm{Cu}_{32} \mathrm{SO}_{4}$ in DMSO-d 6 (OH signals in the $(-25)-(-59) \mathrm{ppm}$ window). 


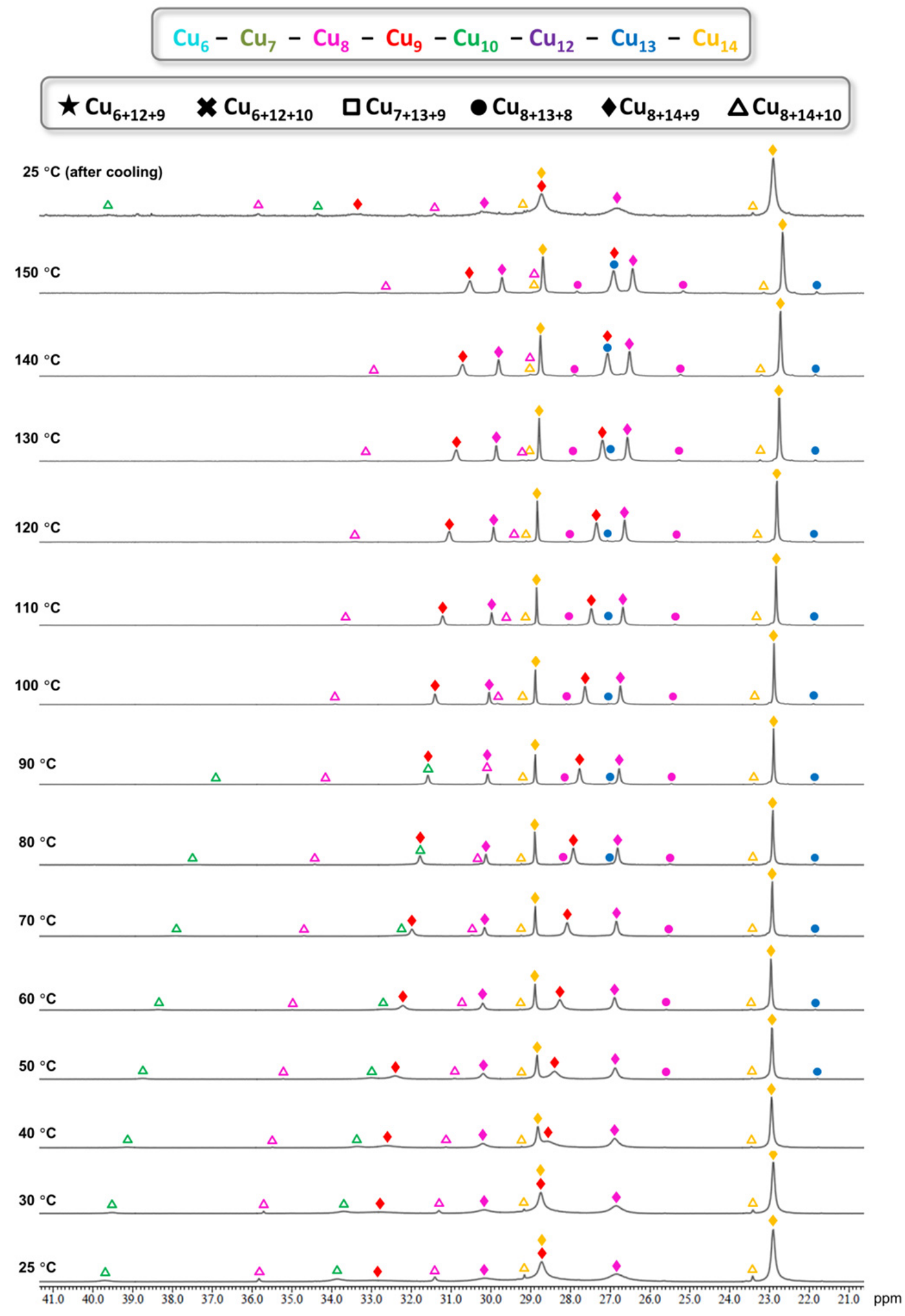

Figure S19. Variable-temperature ${ }^{1} \mathrm{H} N M R$ spectra of $\left(\mathrm{Bu}_{4} \mathrm{~N}\right)_{2}\left[\mathrm{SO}_{4} \subset\{\mathrm{Cu}(\mathrm{OH})(\mathrm{pz})\}_{31}\right]$ with small amounts of $\left(\mathrm{Bu}_{4} \mathrm{~N}\right)_{2}\left[\mathrm{SO}_{4} \subset\{\mathrm{Cu}(\mathrm{OH})(\mathrm{pz})\}_{32}\right]$ in DMSO- $d_{6}$ (pyrazole signals in the 21-41 ppm window). 


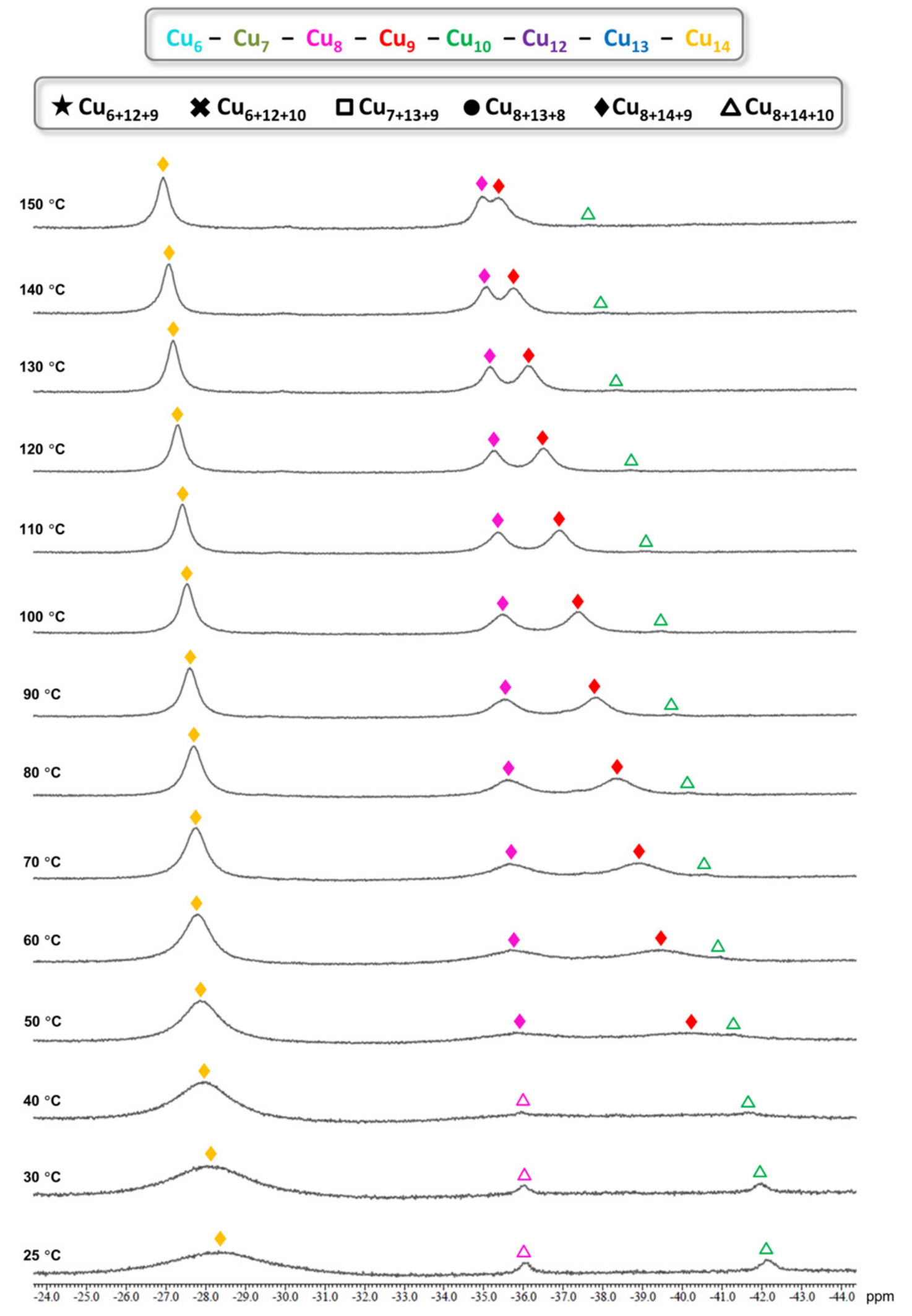

Figure S20. Variable-temperature ${ }^{1} \mathrm{H}$ NMR spectra of $\left(\mathrm{Bu}_{4} \mathrm{~N}\right)_{2}\left[\mathrm{SO}_{4} \subset\{\mathrm{Cu}(\mathrm{OH})(\mathrm{pz})\}_{31}\right]$ with small amounts of $\left(\mathrm{Bu}_{4} \mathrm{~N}\right)_{2}\left[\mathrm{SO}_{4} \subset\{\mathrm{Cu}(\mathrm{OH})(\mathrm{pz})\}_{32}\right]$ in DMSO-d $d_{6}(\mathrm{OH}$ signals in the $(-25)-(-59)$ ppm window). 

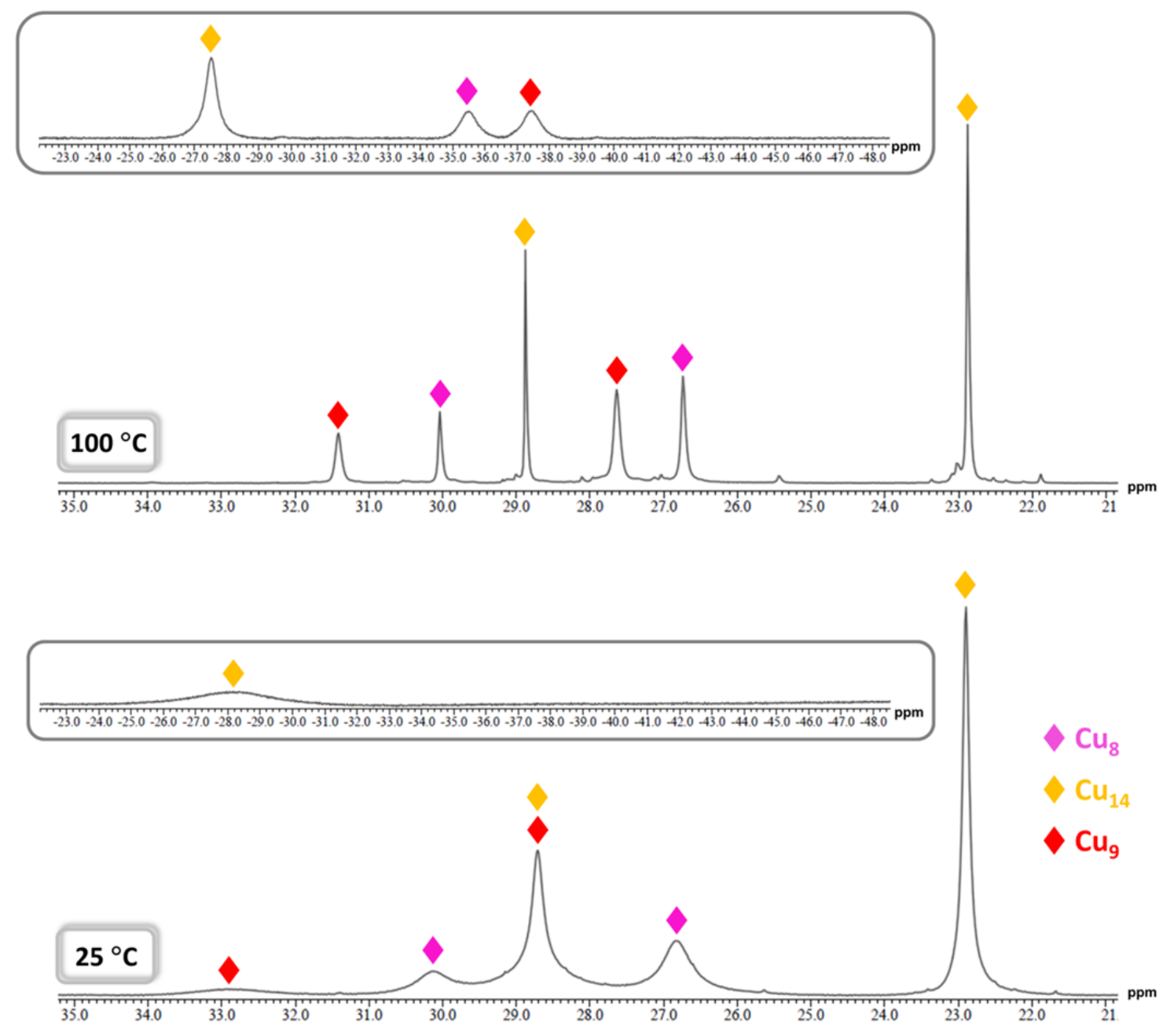

Figure S21. ${ }^{1} \mathrm{H}$ NMR spectra of pure $\left(\mathrm{Bu}_{4} \mathrm{~N}\right)_{2}\left[\mathrm{SO}_{4} \subset\{\mathrm{Cu}(\mathrm{OH})(\mathrm{pz})\}_{31}\right]$ in DMSO- $d_{6}$ at $25^{\circ} \mathrm{C}$ and $100{ }^{\circ} \mathrm{C}$, showing pyrazole proton signals in the $21-35 \mathrm{ppm}$ window. Insets show the corresponding $\mathrm{OH}$ proton signals. Small peaks corresponding to $\mathrm{Cu}_{8+13+8} \mathrm{SO}_{4}$ decomposition product are observed at high temperature. 


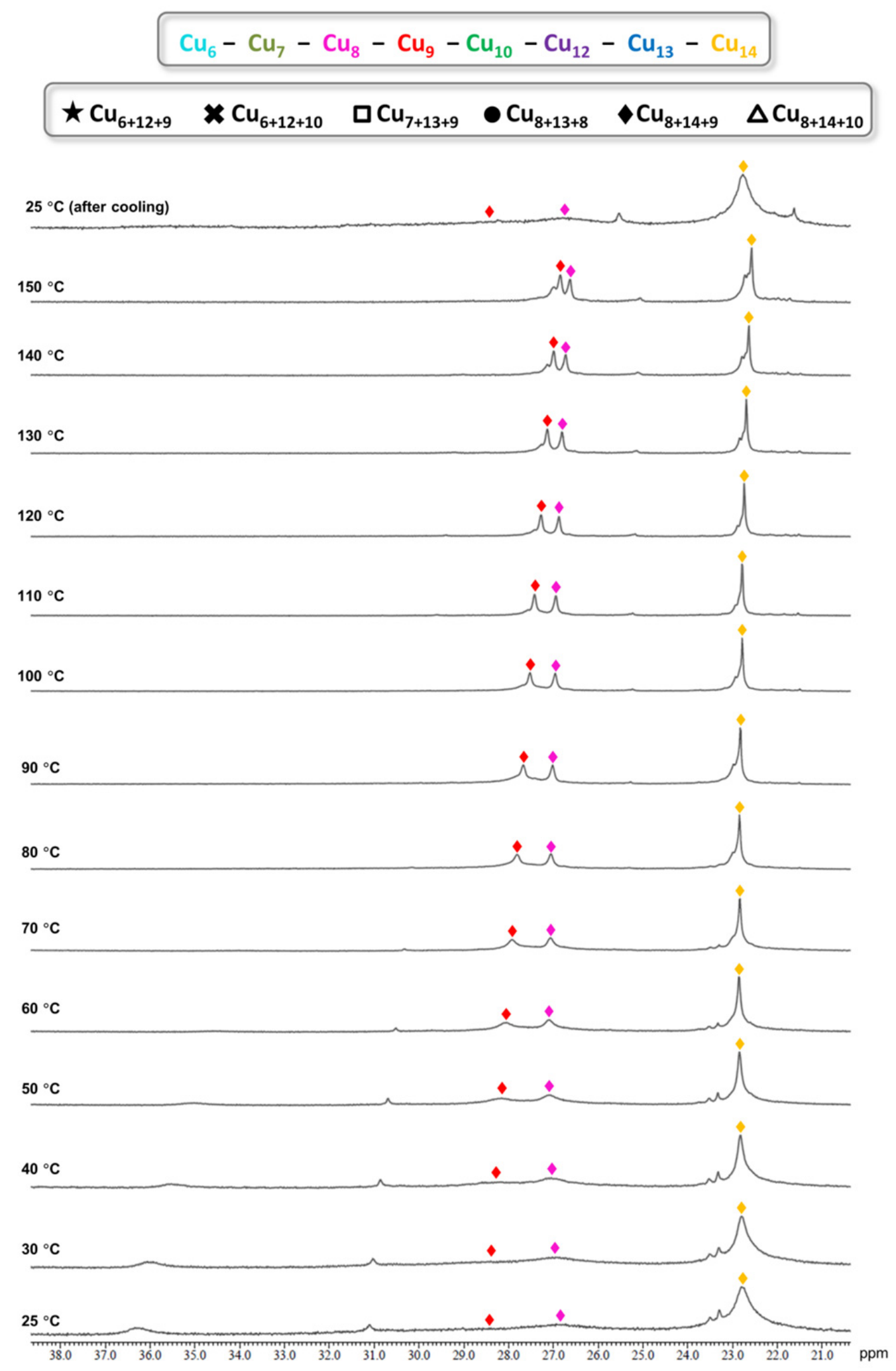

Figure S22. Variable-temperature ${ }^{1} \mathrm{H}$ NMR spectra of $\left(\mathrm{Bu}_{4} \mathrm{~N}\right)_{2}\left[\mathrm{SO}_{4} \subset\{\mathrm{Cu}(\mathrm{OH})(4-\mathrm{Mepz})\}_{31}\right]$ with small amounts of $\left(\mathrm{Bu}_{4} \mathrm{~N}\right)_{2}\left[\mathrm{SO}_{4} \subset\{\mathrm{Cu}(\mathrm{OH})(4-\mathrm{Mepz})\}_{32}\right]$ in DMSO- $d_{6}$ (pyrazole signals in the 21-41 ppm window). 


$$
\frac{\mathrm{Cu}_{6}-\mathrm{Cu}_{7}-\mathrm{Cu}_{8}-\mathrm{Cu}_{9}-\mathrm{Cu}_{10}-\mathrm{Cu}_{12}-\mathrm{Cu}_{13}-\mathrm{Cu}_{14}}{\star \mathrm{Cu}_{6+12+9} \mathrm{CO}_{3} \square \mathrm{Cu}_{7+13+9} \mathrm{CO}_{3} \odot \mathrm{Cu}_{8+13+8} \mathrm{CO}_{3}}
$$

$\boldsymbol{x} \mathrm{Cu}_{6+12+10} \mathrm{SO}_{4} \quad \square \mathrm{Cu}_{7+13+9} \mathrm{SO}_{4} \quad \mathrm{OCu}_{8+13+8} \mathrm{SO}_{4} \quad \diamond \mathrm{Cu}_{8+14+9} \mathrm{SO}_{4} \quad \Delta \mathrm{Cu}_{8+14+10} \mathrm{SO}_{4}$

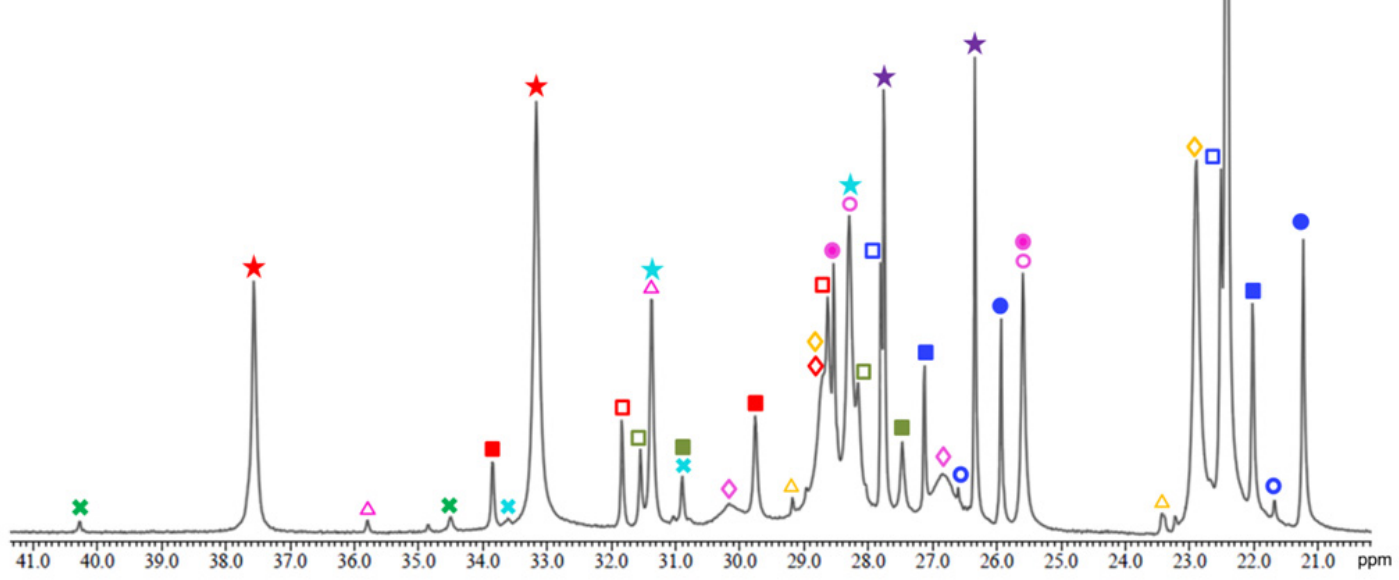

Figure S23. ${ }^{1} \mathrm{H}$ NMR spectrum (in DMSO- $d_{6}$ ) of a mixture of sulfate- and carbonate-incarcerating nanojars with a $\mathrm{SO}_{4}{ }^{2-}: \mathrm{CO}_{3}{ }^{2-}$ molar ratio of $1: 1$ (all reagents soluble in the THF reaction medium), after refluxing the THF solution for 24 hours (pyrazole signals in the 21-41 ppm window). 

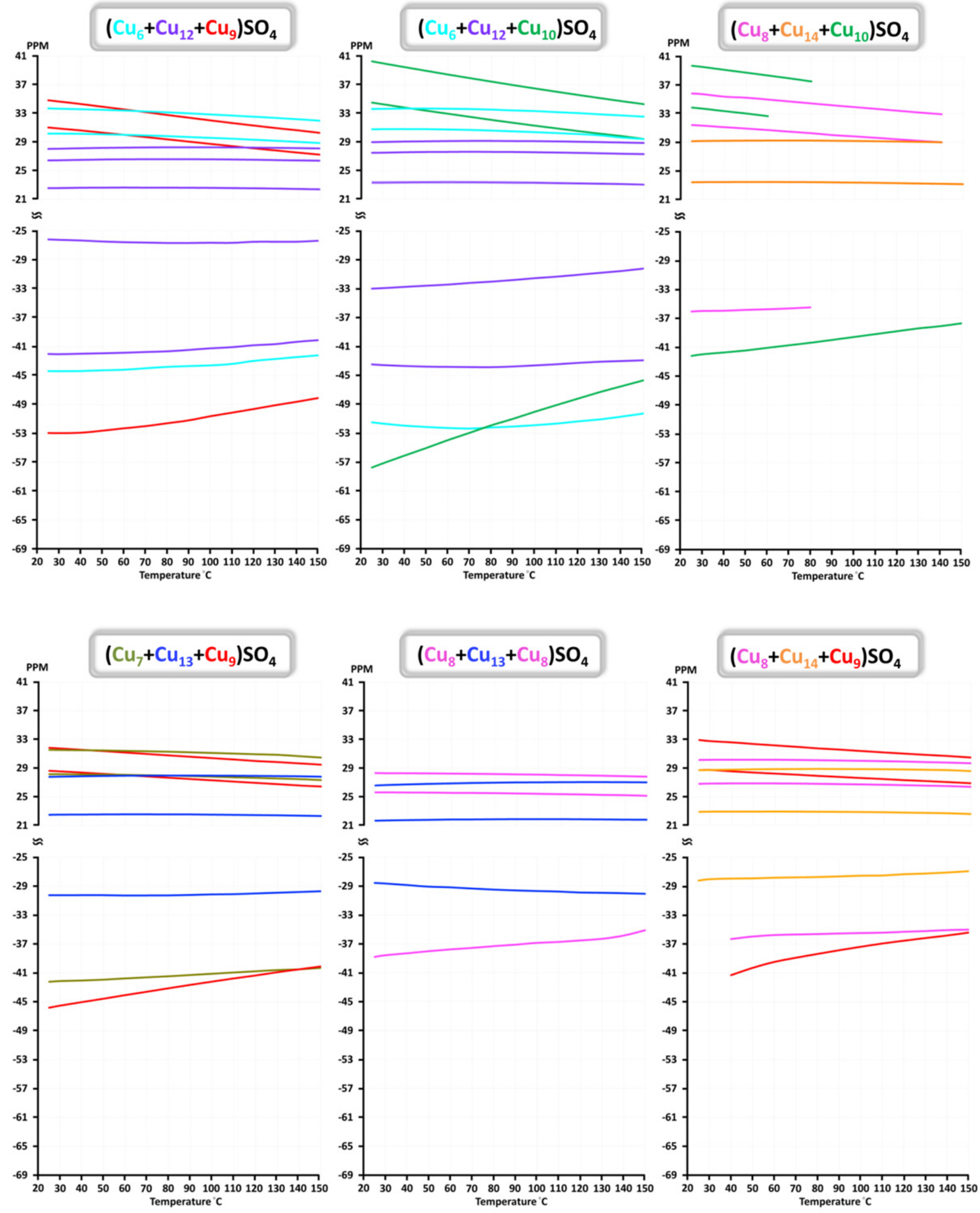

Figure S24. Temperature-dependent variation of the chemical shift of different $\mathrm{Cu}_{\mathrm{n}}$-ring protons in the various sulfate-nanojars in DMSO- $d_{6}$, illustrating the influence of the size of the ring on chemical shift, as well as the difference in chemical shift for the same $\mathrm{Cu}_{\mathrm{n}}$-ring in different nanojars.

S21 


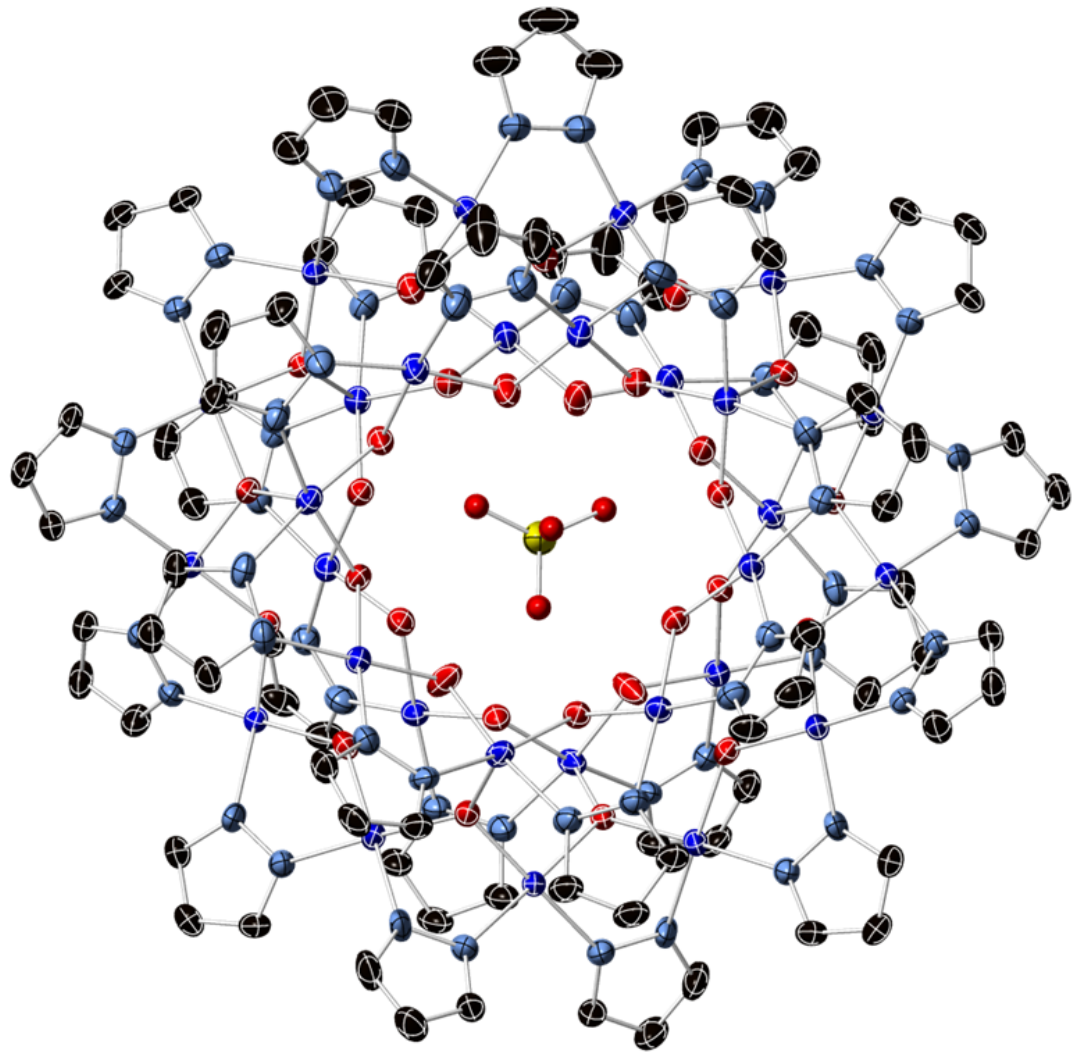

Figure S25. Thermal ellipsoid plot (50\% probability level) of $\left[\mathrm{SO}_{4} \subset\{\mathrm{Cu}(\mathrm{OH})(\mathrm{pz})\}_{8+13+8}\right]^{2-}$ (hydrogen atoms, counterions and solvent molecules not shown). Disordered O-atoms of the sulfate ion are shown isotropic.

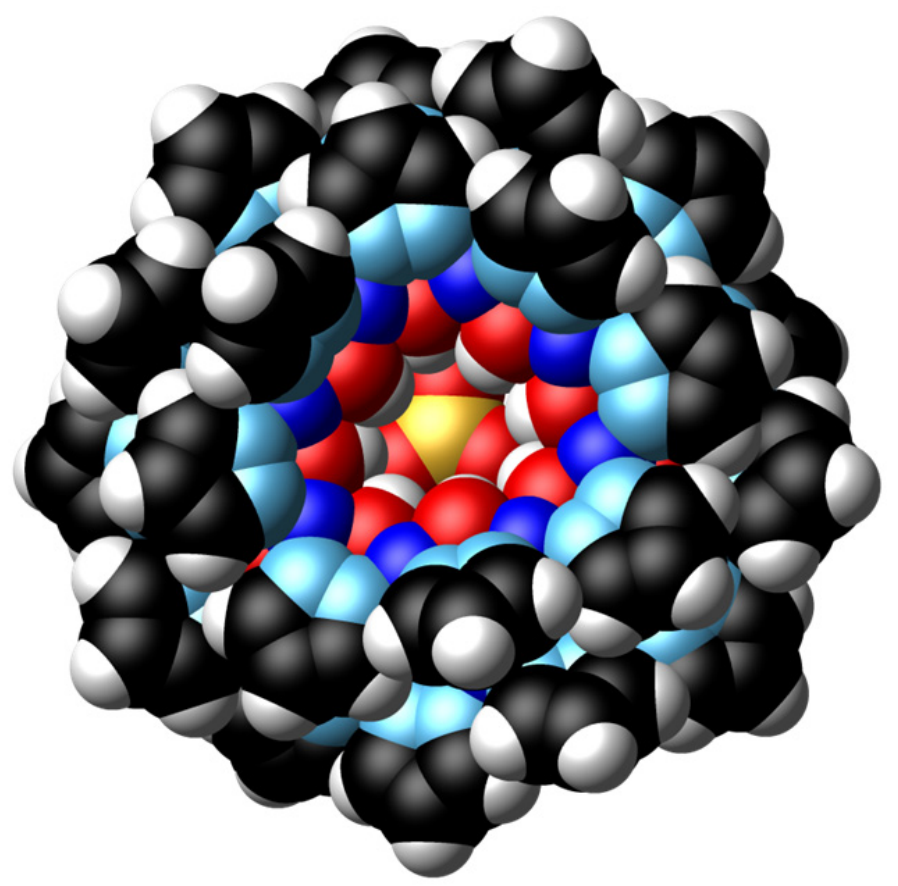

Figure S26. Space-filling representation of $\left[\mathrm{SO}_{4} \subset\{\mathrm{Cu}(\mathrm{OH})(\mathrm{pz})\}_{8+13+8}\right]^{2-}$. 

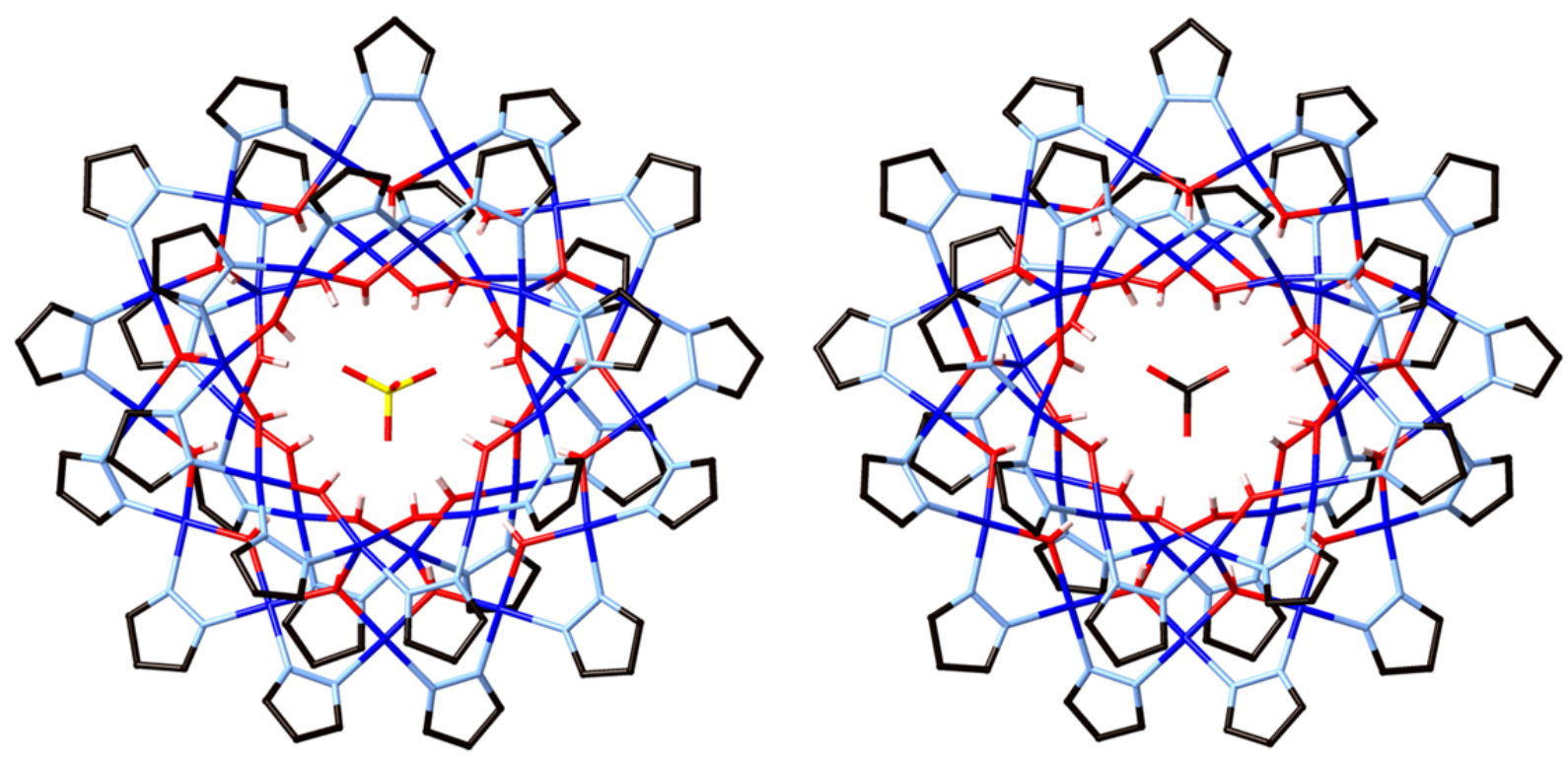

Figure S27. Comparison of the crystal structures of sulfate- and carbonate-incarcerating nanojars $\left[\mathrm{SO}_{4} \subset\{\mathrm{Cu}(\mathrm{OH})(\mathrm{pz})\}_{8+13+8}\right]^{2-}$ and $\left[\mathrm{CO}_{3} \subset\{\mathrm{Cu}(\mathrm{OH})(\mathrm{pz})\}_{8+13+8}\right]^{2-}$.

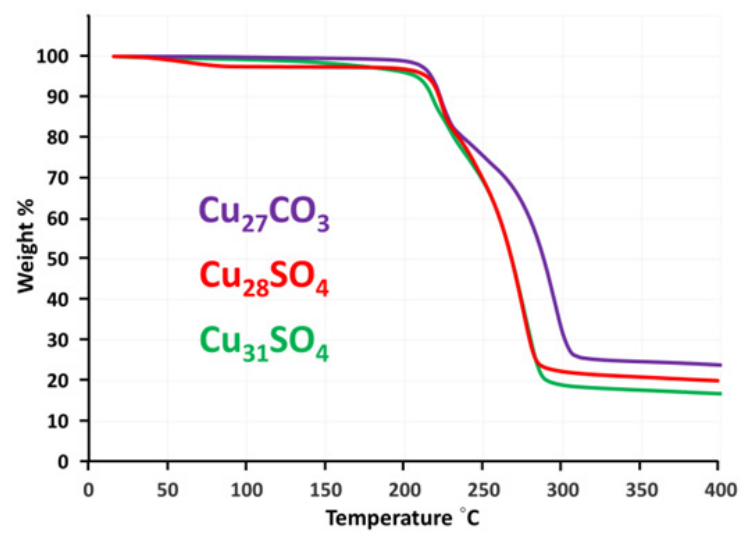

Figure S28. Thermogravimetric curves for the $\mathrm{Cu}_{27} \mathrm{CO}_{3}, \mathrm{Cu}_{28} \mathrm{SO}_{4}$ (with small amounts of $\mathrm{Cu}_{27} \mathrm{SO}_{4}$ ) and $\mathrm{Cu}_{31} \mathrm{SO}_{4}$ (with small amounts of $\mathrm{Cu}_{32} \mathrm{SO}_{4}$ ) heated at a rate of $5{ }^{\circ} \mathrm{C} /$ min under $\mathrm{N}_{2} \cdot \mathrm{Cu}_{28} \mathrm{SO}_{4}$ retains a toluene solvent molecule at room temperature under vacuum, which is lost on heating above $\sim 50{ }^{\circ} \mathrm{C}$ $(1.9 \%)$. 